\title{
Exponentiation for products of Wilson lines within the generating function approach
}

\author{
A.A. Vladimirov \\ Department of Astronomy and Theoretical Physics, Lund University, \\ Sölvegatan 14A, Lund, S 22362 Sweden \\ E-mail: vladimirov.aleksey@gmail.com
}

ABSTRACT: We present the generating function approach to the perturbative exponentiation of correlators of a product of Wilson lines and loops. The exponentiated expression is presented in closed form as an algebraic function of correlators of known operators, which can be seen as a generating function for web diagrams. The expression is naturally split onto two parts: the exponentiation kernel, which accumulates all non-trivial information about web diagrams, and the defect of exponentiation, which reconstructs the matrix exponent and is a function of the exponentiation kernel. The detailed comparison of the presented approach with existing approaches to exponentiation is presented as well. We also give examples of calculations within the generating function exponentiation, namely, we consider different configurations of light-like Wilson lines in the multi-gluon-exchangewebs (MGEW) approximation. Within this approximation the corresponding correlators can be calculated exactly at any order of perturbative expansion by only algebraic manipulations. The MGEW approximation shows violation of the dipole formula for infrared singularities at three-loop order.

Keywords: Wilson, 't Hooft and Polyakov loops, Resummation, QCD, Parton Model

ARXIV EPRINT: 1501.03316 


\section{Contents}

1 Introduction 1

2 Brief review of exponentiation methods 3

3 Generating function approach to exponentiation $\quad 4$

3.1 The foundation of exponentiation 5

$\begin{array}{lll}3.2 & \text { Exponentiation of Wilson lines in QED } & 6\end{array}$

$\begin{array}{lll}3.3 & \text { Exponentiation of real exchanges } & 7\end{array}$

4 Exponentiation of Wilson lines in non-Abelian gauge theories 9

4.1 Wilson line as exponent 9

$\begin{array}{ll}4.2 \text { Matrix exponentiation } & 11\end{array}$

$\begin{array}{lll}4.3 & \text { Structure of MEK } & 14\end{array}$

$\begin{array}{lll}4.4 & \text { Structure of the defect } & 15\end{array}$

5 Application of GF exponentiation $\quad 18$

$\begin{array}{lll}5.1 \text { Cusp at two loops } & 19\end{array}$

5.2 Multiple gluon exchange webs for light-like Wilson lines 23

$\begin{array}{llr}6 & \text { Conclusion } & 27\end{array}$

$\begin{array}{ll}\text { A Operator } V_{n} \text { at low orders } & 29\end{array}$

\section{Introduction}

The dynamics of Wilson lines and loops is responsible for nearly every aspect of gauge theories. Naturally, the correlators of Wilson lines and loops are one of the most attractive objects of theoretical investigations. In this article we present the generating functional approach to the exponentiation of a general correlator of Wilson lines.

The exponentiation of a correlator of Wilson lines is the fundamental property of the perturbative approach. As the name suggests, the exponentiation property allows one to present the perturbative series for the correlator as an exponent. The non-triviality of this statement consists in the special diagrammatic of the exponentiated expression. Namely, only connected diagrams enter the argument of the exponent. Moreover, often the exponentiated diagrams possess some additional non-trivial properties, being a subset of the initial (not exponentiated) series of diagrams. Such an approach can be considered as an exact resummation of the initial diagram series. In this way, the knowledge of the rules for exponentiated diagrammatic significantly simplifies analysis of the Wilson lines correlators within the perturbation theory. 
The exponentiation property for Abelian gauge theories has been understood a long time ago [1]. The exponentiation for a non-Abelian gauge theory is significantly more complicated, and until the recent time, only the exponentiation property for the cusp configuration of Wilson lines and for a Wilson loop was known [2-4]. The exponentiated diagrams for the cusp were called "webs" [2], and were investigated in great details. They play an important role in the description of the strong interaction dynamics. However, nowadays more complex configurations of Wilson lines are interesting. During the last decade several approaches to the exponentiation of a general correlator of Wilson lines were presented [5-8] (the brief review of these approaches is given in section 2).

In this article we present and elaborate details of the exponentiation approach via the generating function, which has been partially presented in [9]. The main object of our interest is the correlator of any number of Wilson lines of any paths and of any group representations

$$
\mathcal{S}_{N}\left(\left\{\gamma_{1}, f_{1}\right\}, \ldots,\left\{\gamma_{N}, f_{N}\right\}\right)=\left\langle\Phi_{\gamma_{1}}^{f_{1}} \times \ldots \times \Phi_{\gamma_{N}}^{f_{N}}\right\rangle
$$

where $\Phi$ is a Wilson line of representation $f$ along the path $\gamma$ :

$$
\Phi_{\gamma}^{f}=P \exp \left(i g \int_{0}^{1} d \tau \dot{\gamma}^{\mu}(\tau) A_{\mu}^{a}(\gamma(\tau)) t_{a}^{[f]}\right),
$$

where $\gamma(\tau)$ parameterizes the path, and $\dot{\gamma}$ is a tangent to the path at point $\tau, t^{[f]}$ are the generators of the gauge group in the representation $f$. We stress that the Wilson line (1.2) is a matrix in the color space, and hence, the correlator (1.1) is a multi-matrix. The disentangling of the matrix structure is the main difficulty of the exponentiation in a non-Abelian gauge theory. In particular, inaccurate treatment of the matrix structure gives rise to the incomplete exponentiated expression in ref. [9]. This issue is corrected in the present article.

The method of exponentiation presented in the article, which we call the generating function exponentiation, is novel and qualitatively differs from the methods presented in the literature. The main feature of the generating function exponentiation is that the argument of exponent is presented in closed form, namely, as a function of correlators of certain operators. This function has the meaning of the generating function for web diagrams.

Technically, the main difference between the generating function exponentiation and other methods of exponentiation consists in the splitting of the problem onto two principally different mathematical tasks: the exponentiation of a scalar operator in a non-Abelian field theory, and the exponentiation of a matrix object. Separately these tasks are effortless. However, together they produce many various diagrams and factors, the straightforward exponentiation of which is a cumbersome work. Therefore, despite many parallels with exponentiation methods presented in literature, we found the final result unique. The detailed explicit comparison of the exponentiation approaches is given in section 5 .

The article is composed as follows. In section 2 we make a brief review of exponentiation methods. The main aim of this section is to introduce a minimal terminology. In 
section 3 we present elementary introduction to the generating function approach to the exponentiation, in particular, we demonstrate the exponentiation in the Abelian gauge theory and the exponentiation of real exchanges. Section 4 is the main section of the article. In this section we present the generating function approach to the non-Abelian exponentiation and derive all the main formulae. Finally, section 5 presents a set of explicit applications of the generating function exponentiation. In particular, in section 5.1 on the example of the two-loop cusp, we perform diagram-by-diagram comparison of the presented approach with existing approaches, while in section 5.2 we demonstrate the effectiveness of the method by evaluating some configurations of light-like Wilson lines in the multi-gluon-exchange-webs approximation.

\section{Brief review of exponentiation methods}

In the paper we present an exponentiation method for a product of Wilson lines and compare it with other exponentiation approaches presented in literature. The methods (the one presented here and those taken from literature) have similar and distinct points. In order to clarify the presentation let us shortly describe the exponentiation methods and introduce necessary terminology.

The most straightforward approach to the exponentiation is to consider the logarithm of the perturbative series. Performing the perturbative expansion of the logarithm and combining diagrams together one obtains the exponentiated perturbative series. Within the composition of diagrams many terms cancel and the remainder is called web diagrams, or webs [2]. We name such an approach to the exponentiation as the diagrammatic exponentiation.

For the cusp and for a Wilson loop the diagrammatic approach gives the following result for the exponentiated series of diagrams. The color factor of the diagrams (of the non-exponentiated series) should be replaced by the modified color factors [3, 4],

$$
\operatorname{tr}\langle\Phi\rangle=\sum_{d} C(d) \mathcal{F}(d)=\exp \left(\sum_{d} \tilde{C}(d) \mathcal{F}(d)\right),
$$

where $C(d)$ and $\mathcal{F}(d)$ are the color factor and the kinematical part of a diagram $d$, respectively. The modified color factor $\tilde{C}$ is obtained by the recursive procedure

$$
\tilde{C}(d)=C(d)-\sum_{d^{\prime}} \prod_{w \in d^{\prime}} \tilde{C}(w)
$$

where the sum runs over all decompositions of $d$ into two-Wilson-lines-irreducible subgraphs $w$ (webs). In the consequence of the expression (2.2) only the two-Wilson-lines-irreducible diagrams have non-zero modified color factors. These diagrams form the set of webs for the cusp, or for the Wilson loop.

For a general configuration of Wilson lines the diagrammatic exponentiation has been considered in ref. [7]. It has been shown that for the general case no simple selection criterium exists, and all diagrammatic topologies enter the exponentiated series. 
Another method of exponentiation was suggested in ref. [5] and elaborated in $[6,8,10]$. The method is based on the replica trick and hence, we name it as the replica exponentiation.

Briefly, the course of the replica exponentiation is the following. In the first step, the correlator of (replica-ordered) Wilson lines is considered in the replicated theory, i.e. the theory that consists of $N_{\text {rep }}$ copies of the original theory (replicas). The obtained diagrammatic expansion depends on $N_{\text {rep }}$. In the second step, the terms proportional to $N_{\text {rep }}$ are taken, and the rest terms are discarded. These terms form the argument of the exponent in the original theory. The resulting expression can be presented in the following form

$$
\langle\Phi \times \Phi \ldots \times \Phi\rangle_{I J}=\exp \left(\sum_{d, d^{\prime}} \mathcal{F}(d) R_{d, d^{\prime}} C\left(d^{\prime}\right)\right)_{I J},
$$

where $R$ is the web-mixing matrix $[6,10]$, and $I$ and $J$ are the color multi-indices. The product $R C$ generalizes the concept of the modified color factor of the color-singlet case (2.2).

The replica exponentiation gives deeper theoretical understanding of the exponentiation procedure, and allows one to make certain general conclusions about webs. For example, using the replica exponentiation it has been shown that color factors of the exponentiated expression contain only the color connected combinations of diagrams [8]. Recently, the method has been improved by the effective vertex formalism [8], and in this form it was used for analysis of particular multi-loop webs [11].

The disadvantage of the replica method is that it does not present any universal expression for the series of web diagrams. The web-mixing matrix should be calculated at every order of the perturbative expression independently, what makes any general consideration difficult. In this aspect, the replica exponentiation resembles the diagrammatic exponentiation for the product of Wilson lines [7] (for explicit comparison see the appendix of ref. [10]).

In this article we present a novel exponentiation approach. The main distinctive feature of the approach is the presentation of the exponentiated series in closed form, namely, as a function of correlators of known operators. This function can be interpreted as a generating function for web diagrams [9]. Thus, we name the presented method as the exponentiation via generating function or, for shortness, GF exponentiation.

Although GF exponentiation is obtained independently from the diagrammatic exponentiation and the replica exponentiation, these approaches have many common points. GF exponentiation can be viewed as a union of both approaches. As we show later, the generating function consists of two parts. One of those can be easily traced in the replica method (in its formulation via effective operators [8]), while another is closely related to the diagrammatic approach. However, in contrast to the diagrammatic and the replica exponentiations, the GF exponentiation has a visual and simple expression.

\section{Generating function approach to exponentiation}

In this section we discuss the basics of GF exponentiation. The material of the section is somewhat trivial and generally can be collected from textbooks on quantum field theory. 
Therefore, the main aim of this section is not to present a new material, but to introduce notations, and the general way of approach.

We also demonstrate GF exponentiation in Abelian gauge theory. This presentation serves as a pedagogical example, which is later generalized on the non-Abelian case. The presentation of this part closely follows the presentation in ref. [9].

In the last subsection, we briefly discuss the generalization of GF exponentiation on the correlators with real exchanges, which are very important in practice.

\subsection{The foundation of exponentiation}

In ref. [9] it was shown that the quantum average of any operator, which has the form of an exponent of another operator, can be presented as an exponent of the series of connected diagrams. In fact, this statement is a reformulation of the famous relation between the partition function and the series of connected diagrams (3.2). The relation holds in any quantum field theory, therefore, in this section we use maximally abstract notation, denoting all fields of a theory as $A$, and do not specify their quantum numbers.

The partition function of a quantum theory reads

$$
Z[J]=\int D A e^{S[A]+\int d x J(x) O(x)},
$$

where $O(x)$ is a composite operator, $J(x)$ is the source, and $S[A]$ is the action of theory. The diagrammatic expansion of $Z[J]$ consists of all possible Feynman diagrams connected and disconnected, with or without insertions of operator $O$. The logarithm of the partition function

$$
W[J]=\ln \frac{Z[J]}{Z[0]},
$$

has the meaning of the generating function for the Green functions of operators $O$. Diagrammatically, it is given by only connected diagrams with insertions of operator $O$. In the case of many sources, $Z\left[J_{1}, J_{2}, \ldots\right]$, the corresponding generating function $W\left[J_{1}, J_{2}, \ldots\right]$ is given by all connected diagrams with all possible insertions of operators $O_{1}, O_{2}$, etc.

Let us consider an operator of the form

$$
O[A]=\exp \left(\int d x M(x) \mathcal{Y}[A]\right),
$$

where $M(x)$ is some classical field and $\mathcal{Y}[A]$ is a composite operator of fields $A$. According to the definition of the quantum average one can consider the vacuum matrix element of the operator (3.3) as the partition function evaluated on the "classical sources" $M(x)$,

$$
\langle T O[A]\rangle=\frac{1}{Z[0]} \int D A e^{S[A]} e^{\int d x M(x) \mathcal{Y}(x)}=\frac{Z[M]}{Z[0]} .
$$

The T-ordering on the left-hand-side of eq. (3.4) allows us to apply the functional integration quantization. For brevity, in the following we do not explicitly denote T-ordering, assuming it for every quantum average. Exception is made only in section 3.3, where we discuss the quantum averages of operators without T-ordering. 
The matrix element (3.4) can be presented as an exponent of the generating function (3.2). By definition, the generating function can be presented as a series of correlators

$$
\begin{aligned}
\langle O[A]\rangle=e^{W[M]} & \\
=\exp \left(\int\right. & d x M(x)\langle\mathcal{Y}(x)\rangle+\frac{1}{2} \int d x_{1,2} M\left(x_{1}\right) M\left(x_{2}\right)\left\langle\mathcal{Y}\left(x_{1}\right) \mathcal{Y}\left(x_{2}\right)\right\rangle \\
& \left.+\frac{1}{3 !} \int d x_{1,2,3} M\left(x_{1}\right) M\left(x_{2}\right) M\left(x_{3}\right)\left\langle\mathcal{Y}\left(x_{1}\right) \mathcal{Y}\left(x_{2}\right) \mathcal{Y}\left(x_{3}\right)\right\rangle+\ldots\right),
\end{aligned}
$$

where the factorial coefficients are the symmetry coefficients resulting from the permutation symmetry of the correlators. Thus, the exponentiated series for the operator $O[A]$ is given by only connected diagrams with arbitrary number of operators $\mathcal{Y}$ convoluted with the "classical sources" $M(x)$.

The fact that $W[M]$ is given solely by connected diagrams does not imply that the original series for $\langle O\rangle$ contains disconnected diagrams. Both series contain only connected diagrams, but connected to different operator vertices. Since, the operators $\mathcal{Y}$ and $O$ can have very different properties, the series of diagrams contributing to $\langle O\rangle$ can relate to the series of diagrams contributing to $W[M]$ in a very non-trivial way. The case of non-Abelian exponentiation, the main subject of this article, is an example of such a non-trivial relation.

Exponentiation of the perturbative series for an operator of the type (3.3) is the fundamental property of the perturbative expansion. It is founded only on the relations between the symmetry coefficients of various Feynman diagrams. In its own turn, the later is the consequence of the perturbative approach to functional integration, i.e. the property of the expansion of the action exponent around its Gaussian part. It is important to mention that the structure of the propagator does not affect the symmetry coefficients and, thus, does not influence on the exponentiation of the diagrams.

\subsection{Exponentiation of Wilson lines in QED}

Let us demonstrate the application of GF exponentiation of the Wilson line in an Abelian gauge theory (QED).

The starting point of the approach is to present the operator, namely the Wilson line, in the form (3.3). In QED the path-ordered exponent (1.2) is equal to the usual exponent,

$$
\Phi_{\gamma}=\exp \left(i g \int_{0}^{1} d \tau \dot{\gamma}^{\mu}(\tau) A_{\mu}(\gamma(\tau))\right)
$$

Comparing this expression with eq. (3.3) we find that the operator $\mathcal{Y}(x)$ is just the field $A_{\mu}(x)$, while the source $M(x)$ is a classical source of radiation,

$$
M^{\mu}(x)=i g \int_{0}^{1} d \tau \dot{\gamma}(\tau) \delta(\gamma(\tau)-x)
$$

Evaluating the expression (3.5) with the sources (3.7) we obtain the exponentiated expression

$$
\begin{aligned}
\left\langle\Phi_{\gamma}\right\rangle= & \exp \left(\frac{-g^{2}}{2 !} \int_{0}^{1} d \tau_{1} \int_{0}^{1} d \tau_{2} \dot{\gamma}^{\mu_{1}}\left(\tau_{1}\right) \dot{\gamma}^{\mu_{2}}\left(\tau_{2}\right)\left\langle A_{\mu_{1}}\left(\gamma\left(\tau_{1}\right)\right) A_{\mu_{2}}\left(\gamma\left(\tau_{2}\right)\right)\right\rangle\right. \\
& \left.+\frac{g^{4}}{4 !}\left(\prod_{i=1}^{4} \int_{0}^{1} d \tau_{i} \dot{\gamma}^{\mu_{i}}\left(\tau_{i}\right)\right)\left\langle A_{\mu_{1}}\left(\gamma\left(\tau_{1}\right)\right) A_{\mu_{2}}\left(\gamma\left(\tau_{2}\right)\right) A_{\mu_{3}}\left(\gamma\left(\tau_{3}\right)\right) A_{\mu_{4}}\left(\gamma\left(\tau_{4}\right)\right)\right\rangle+\ldots\right)
\end{aligned}
$$


where the correlators with odd number of photons are omitted in consequence of Furry's theorem. Diagrammatically the argument of the exponent is given by connected diagrams with an arbitrary number of external photons positioned on the path of the Wilson line.

The expression (3.8) is already suitable for the further consideration, however, it can be rewritten in the more traditional form. Indeed, using the symmetry of the multi-photon correlators under the permutations of fields we combine multiple sources to a single pathordered source,

$$
\begin{aligned}
\left\langle\Phi_{\gamma}\right\rangle= & \exp \left(-g^{2} \int_{0}^{1} d \tau_{1} \int_{\tau_{1}}^{1} d \tau_{2} \dot{\gamma}^{\mu_{1}}\left(\tau_{1}\right) \dot{\gamma}^{\mu_{2}}\left(\tau_{2}\right)\left\langle A_{\mu_{1}}\left(\gamma\left(\tau_{1}\right)\right) A_{\mu_{2}}\left(\gamma\left(\tau_{2}\right)\right)\right\rangle\right. \\
& +g^{4} \int_{0}^{1} \int_{\tau_{1}}^{1} \int_{\tau_{2}}^{1} \int_{\tau_{3}}^{1}\left(\prod_{i=1}^{4} d \tau_{i} \dot{\gamma}^{\mu_{i}}\left(\tau_{i}\right)\right) \\
& \left.\times\left\langle A_{\mu_{1}}\left(\gamma\left(\tau_{1}\right)\right) A_{\mu_{2}}\left(\gamma\left(\tau_{2}\right)\right) A_{\mu_{3}}\left(\gamma\left(\tau_{3}\right)\right) A_{\mu_{4}}\left(\gamma\left(\tau_{4}\right)\right)\right\rangle+\ldots\right)
\end{aligned}
$$

The symmetry coefficients of (3.8) are canceled by factorial multipliers resulting from the ordering procedure. The expression (3.9) has the common form of the exponent of all completely connected (connected in the absence of Wilson line) diagrams with unity symmetry coefficients.

We stress that within GF exponentiation the contour of a Wilson line plays no role. Therefore, the exponentiation property holds for any contours, including cusped, selfcrossed and disconnected. However, for the contours with singularities it is convenient to introduce independent sources for every individual smooth segment. This allows one to reveal the influence of the contour singularities on the diagrams explicitly.

\subsection{Exponentiation of real exchanges}

Diagrams with real particles exchanges play an important role in applications of quantum field theory, especially, in the consideration of final state interactions of hard processes. Typically, on the operator level the real exchanges appear as an insertion of the complete set of asymptotic states. Alternatively such operators can be given by the (usual) product of T-ordered operators

$$
\left\langle T O_{1}[A] \sum_{X} \mid X\right\rangle\left\langle X \mid T O_{2}[A]\right\rangle=\left\langle\left(T O_{1}[A]\right)\left(T O_{2}[A]\right)\right\rangle .
$$

On the diagrammatic level it implies that diagrams describing (3.10) contain two parts. The interactions inside these parts are presented by the Feynman propagators $\Delta_{\mathrm{F}}$, while the interactions between these two parts are presented by the positive-frequency part of the Pauli-Jordan function, $\Delta^{(+)}$.

We remind that the standard functional integration approach assumes the consideration of only T-ordered operators. The diagrammatic expansion of the functional integral can be expressed via the differential reduction exponent (see [12])

$$
\langle T O[A]\rangle=\left.\frac{1}{Z[0]} e^{\left(\int \frac{d x d x^{\prime}}{2} \frac{\delta}{\delta A(x)} \Delta_{\mathrm{F}}\left(x, x^{\prime}\right) \frac{\delta}{\delta A\left(x^{\prime}\right)}\right)} O[A] e^{S_{\mathrm{int}}[A]}\right|_{A=0}
$$


where $\Delta_{\mathrm{F}}\left(x, x^{\prime}\right)$ is the Feynman propagator, $S_{\text {int }}$ is the interaction part of the action. In the reduction exponent (3.11) the T-ordering of operator is reflected through the Feynman propagator.

In fact, the reduction exponent is the general form of any diagrammatic expansion, but not only the T-ordered as it follows from the functional integral. In order to obtain the diagrammatic expansion for (3.10) we can adjust every operator to an independent copy of a quantum field theory. In this case, the virtual interactions are generated independently by copies of the functional integral. The real particle exchanges between these copies can be added to diagrammatic by the extra reduction exponent with a real propagator. Formally, we have

$$
\begin{aligned}
\left\langle\left(T O_{1}[A]\right)\left(T O_{2}[A]\right)\right\rangle= & \frac{1}{Z^{2}[0]} e^{\left(\int \frac{\delta}{\delta A_{1}} \Delta^{(+)} \frac{\delta}{\delta A_{2}}\right)}\left(e^{\left(\frac{1}{2} \int \frac{\delta}{\delta A_{1}} \Delta_{\mathrm{F}} \frac{\delta}{\delta A_{1}}\right)} O_{1}\left[A_{1}\right] e^{S_{\mathrm{int}}\left[A_{1}\right]}\right) \\
& \times\left.\left(e^{\left(\frac{1}{2} \int \frac{\delta}{\delta A_{2}} \Delta_{\mathrm{F}} \frac{\delta}{\delta A_{2}}\right)} O_{2}\left[A_{2}\right] e^{S_{\mathrm{int}}\left[A_{2}\right]}\right)\right|_{A_{1,2}=0},
\end{aligned}
$$

where we omit the arguments of fields for brevity. In this way, the product of T-ordered operators is presented as a T-ordered product of operators in the modified theory,

$$
\begin{aligned}
\left\langle\left(T O_{1}[A]\right)\left(T O_{2}[A]\right)\right\rangle & =\left\langle T O_{1}[A] O_{2}[A]\right\rangle_{\bmod } \\
& =\frac{1}{Z_{\bmod }[0]} \int D A_{1} D A_{2} O_{1}\left[A_{1}\right] O_{2}\left[A_{2}\right] e^{S\left[A_{1}\right]+S\left[A_{2}\right]+\int A_{1} \Delta^{(+)} A_{2}}
\end{aligned}
$$

Such a trick has been applied in ref. [13] for calculation of Drell-Yan soft factor.

Let us consider two T-ordered operators of the form (3.3). In the course of GF exponentiation we have the chain of equalities

$$
\left\langle\left(T O_{1}[A]\right)\left(T O_{2}[A]\right)\right\rangle=\left\langle T e^{\int M_{1} \mathcal{Y}_{1}\left[A_{1}\right]+\int M_{2} \mathcal{Y}_{2}\left[A_{2}\right]}\right\rangle_{\bmod }=e^{W_{\bmod }\left[M_{1}, M_{2}\right]} .
$$

The modified argument of the exponent reads

$$
\begin{aligned}
W_{\text {mod }}\left[M_{1}, M_{2}\right]= & \int d x\left(M_{1}(x)\left\langle T \mathcal{Y}_{1}\left[A_{1}\right]\right\rangle_{\bmod }+M_{2}(x)\left\langle T \mathcal{Y}_{2}\left[A_{2}\right]\right\rangle_{\bmod }\right) \\
+ & \int d x_{1,2}\left(\frac{1}{2} M_{1}\left(x_{1}\right) M_{1}\left(x_{2}\right)\left\langle T \mathcal{Y}_{1}\left[A_{1}\right] \mathcal{Y}_{1}\left[A_{1}\right]\right\rangle_{\bmod }\right. \\
& +\frac{1}{2} M_{2}\left(x_{1}\right) M_{2}\left(x_{2}\right)\left\langle T \mathcal{Y}_{2}\left[A_{2}\right] \mathcal{Y}_{2}\left[A_{2}\right]\right\rangle_{\bmod } \\
& \left.+M_{1}\left(x_{1}\right) M_{2}\left(x_{2}\right)\left\langle T \mathcal{Y}_{1}\left[A_{1}\right] \mathcal{Y}_{2}\left[A_{2}\right]\right\rangle_{\bmod }\right)+\ldots,
\end{aligned}
$$

where the arguments of operators are omitted for brevity. 
Any expression in the modified theory can be transformed back to the usual theory using the rule (3.13). Therefore, eq. (3.15) transforms to

$$
\begin{aligned}
W\left[M_{1}, M_{2}\right]= & \int d x\left(M_{1}(x)\left\langle T \mathcal{Y}_{1}\right\rangle+M_{2}(x)\left\langle T \mathcal{Y}_{2}\right\rangle\right) \\
& +\int d x_{1,2}\left(\frac{1}{2} M_{1}\left(x_{1}\right) M_{1}\left(x_{2}\right)\left\langle T \mathcal{Y}_{1} \mathcal{Y}_{1}\right\rangle+\frac{1}{2} M_{2}\left(x_{1}\right) M_{2}\left(x_{2}\right)\left\langle T \mathcal{Y}_{2} \mathcal{Y}_{2}\right\rangle\right. \\
& \left.\quad+M_{1}\left(x_{1}\right) M_{2}\left(x_{2}\right)\left\langle\left(T \mathcal{Y}_{1}\right)\left(T \mathcal{Y}_{2}\right)\right\rangle\right)+\ldots
\end{aligned}
$$

where the arguments of operators are omitted for brevity. We conclude that the diagrams with real exchanges exponentiate in the same way as the usual virtual diagrams.

\section{Exponentiation of Wilson lines in non-Abelian gauge theories}

The exponentiation in non-Abelian gauge theories is a more involved problem than the exponentiation in Abelian ones. In comparison to QED there are two sources of complication. The first complication comes from the involved form of operators $\mathcal{Y}$. The second complication comes from the (color-)matrix structure of the non-Abelian Wilson line. The structure of operators $\mathcal{Y}$ and the generating function was elaborated in ref. [9]. However, in ref. [9] the matrix issues of the non-Abelian exponentiation were missed. In this section we present a detailed derivation of the non-Abelian exponentiation.

We remind that within the framework of GF exponentiation the only important information is the path-order of gauge fields, but not their coordinates. The term Wilson line, which is used all over the article, denotes rather a Wilson curve on arbitrary path, than a straight Wilson line, as it may be suggested from the term. Generally, the path can be arbitrary difficult, or even non-analytical. However, we suppose that every individual Wilson line is smooth.

\subsection{Wilson line as exponent}

The starting point of GF exponentiation is to present the Wilson line (1.2) in the form (3.3). For this purpose we use the following exponential representation for the Wilson line,

$$
\begin{aligned}
\Phi_{\gamma}^{f}= & \exp \left\{i g \int_{0}^{1} A_{0}+\sum_{s=1}^{\infty}(i g)^{s+1} \sum_{k=1}^{s} \frac{(-1)^{k}}{k+1}\right. \\
& \left.\times \sum_{\substack{j_{1}+\ldots+j_{k}=s \\
j_{i} \geqslant 1}} \int_{0}^{1}\left(\int_{0}^{\tau} \ldots \int_{0}^{\tau_{j_{1}-1}} \operatorname{ad}_{A_{1}} \ldots \operatorname{ad}_{A_{j_{1}}}\right) \ldots\left(\int_{0}^{\tau} \ldots \int_{0}^{\tau_{j_{k}-1}} \operatorname{ad}_{A_{1}} \ldots \operatorname{ad}_{A_{j_{k}}}\right) A_{0}\right\},
\end{aligned}
$$

where $A_{i}=\dot{\gamma}^{\mu}\left(\tau_{i}\right) \hat{A}_{\mu}\left(\gamma\left(\tau_{i}\right)\right) d \tau_{i}$ and $\tau_{0}=\tau$, with $\hat{A}_{\mu}(x)=t_{a}^{[f]} A_{\mu}^{a}(x)$. The operator $\operatorname{ad}_{A}$ is defined $\operatorname{as~}_{A} X=[A, X]$. The detailed derivation of this relation can be found in ref. [14]

The representation (4.1) reveals several important properties. The main of them is that the operator $\mathcal{Y}$ consists only of completely nested commutators of generators of gauge 
fields. It leads to the color-connectivity of webs, the property that has been the defining property of the webs for many years starting from [3].

The completely nested commutator structure of the operators also implies universality of the representation (4.1) for Wilson lines of different group representation. Indeed, the commutator of generators is proportional to the structure constant $\left[t_{a}^{[f]}, t_{b}^{[f]}\right]=i f_{a b c} t_{c}^{[f]}$. The structure constant is independent of group representation. Therefore, the representation dependence of the operator $\mathcal{Y}$ is concentrated in a single generator, which can be moved out of the operator. The equation (4.1) takes the form

$$
\Phi_{\gamma}^{f}=\exp \left(t_{a}^{[f]} V_{\gamma}^{a}\right)
$$

where $V_{\gamma}^{a}$ are operators independent on the representation of the Wilson line. Here the definition of the operator $V$ is slightly different from the corresponding definition in ref. [9], where the corresponding operator was defined with the integration over $\tau_{0}$ removed. The representation (4.2) also has an advantage that all the matrix structure of the Wilson line is concentrated in the single generator $t_{a}^{[f]}$.

In the case of multiple Wilson lines, it is convenient to merge all Wilson lines to a single exponent. With this purpose we demand that every separate Wilson line acts in a separate matrix space. Then the whole composition of Wilson lines is a matrix of the reducible representation $f_{1} \otimes f_{2} \otimes \ldots \otimes f_{N}$, where $f_{i}$ is the representation of $i$ 'th Wilson line. This trick is often used to simplify the consideration of web diagrams, see e.g. [5, 7]. In order to simplify the notation we introduce the generator in this space with appropriate numbering

$$
T_{A}= \begin{cases}t_{A}^{\left[f_{1}\right]} \otimes 1 \otimes \ldots \otimes 1, & A=1, \ldots, \operatorname{dim}_{\mathrm{G}}, \\ \mathbf{1} \otimes t_{A}^{\left[f_{2}\right]} \otimes \ldots \otimes \mathbf{1}, & A=\operatorname{dim}_{\mathrm{G}}+1, \ldots, 2 \operatorname{dim}_{\mathrm{G}}, \\ \ldots & \\ 1 \otimes 1 \otimes \ldots \otimes t_{A}^{\left[f_{N}\right]}, & A=(N-1) \operatorname{dim}_{\mathrm{G}}+1, \ldots, N \operatorname{dim}_{\mathrm{G}},\end{cases}
$$

where $\operatorname{dim}_{\mathrm{G}}$ is the dimension of the gauge group or, equivalently, the number of generators. We adopt the convention that the labels in the joined space are capitalized, while the labels in the irreducible spaces are denoted by the lowercase letters. Simultaneously, the path dependence of the operator $V_{\gamma}^{a}$ is adjusted to the corresponding sector of label $A$,

$$
V^{A}= \begin{cases}V_{\gamma_{1}}^{A}, & A=1, \ldots, \operatorname{dim}_{\mathrm{G}}, \\ V_{\gamma_{2}}^{A}, & A=\operatorname{dim}_{\mathrm{G}}+1, \ldots, 2 \operatorname{dim}_{\mathrm{G}}, \\ \ldots & A=(N-1) \operatorname{dim}_{\mathrm{G}}+1, \ldots, N \operatorname{dim}_{\mathrm{G}} .\end{cases}
$$

In this representation the product of several Wilson lines reads

$$
\left(\Phi_{\gamma_{1}}^{f_{1}}\right)_{i_{1} j_{1}} \times\left(\Phi_{\gamma_{2}}^{f_{2}}\right)_{i_{2} j_{2}} \times \ldots \times\left(\Phi_{\gamma_{N}}^{f_{N}}\right)_{i_{N} j_{N}}=\Phi_{I J}=\left(\exp T_{A} V^{A}\right)_{I J}
$$

where $I$ and $J$ are indices of the joined matrix space. 
The representation (4.5) resembles the required form (3.3), where generators $T_{A}$ play the role of the matrix sources for operators $V^{A}$. For the general discussion of GF exponentiation it is convenient to keep the infinite sum of operators (4.1) as a single object $V^{A}$. While for the perturbative analysis and practical applications it is convenient to split the operator $V^{A}$ on individual terms of a fixed perturbative order,

$$
V^{A}=\sum_{n=1}^{\infty} V_{n}^{A}
$$

where $V_{n} \sim g^{n}$. The expressions for the first few operators $V_{n}$, as well as, Feynman rules for them are presented in the appendix A.

\subsection{Matrix exponentiation}

According to the general discussion of section 3.1, the connected diagrams with all possible insertions of the operators $V^{A}$ are the only diagrams contributing to the exponent. However, in the case of non-Abelian gauge theory it is not entirely correct. The point is that in the non-Abelian gauge theories the operators in diagrams are caused by the matrix sources $T^{A}$. This complication spoils the usual relations between connected and disconnected diagrams, and prevent the straightforward exponentiation.

The direct way to bring the diagrammatic series into the exponentiated form is to consider its logarithm. The perturbative expansion of the logarithm (of the perturbative series) mixes up the diagrams such that it is very difficult to find out on a general level, which parts of diagrams cancel and which do not. Therefore, this way necessarily leads us to the analysis of individual Feynman diagrams, the procedure which that we try to avoid. This approach was elaborated in ref. [7], and indeed it appears to be not efficient.

In order to solve the matrix complications of the non-Abelian exponentiation in the most efficient way, we split the consideration onto two independent tasks. First, we consider the exponentiation of a scalar version of the non-Abelian Wilson line. That grants us the most promising starting point for the exponentiated diagrammatic. Second, we generalize the scalar version of Wilson line and its exponentiated expression on the matrix form. In this way we obtain the complete matrix exponentiated expression without lost of efficiency of GF exponentiation.

The first point of our program is to consider a scalar operator

$$
\phi=\exp \left(M^{A} V_{A}\right),
$$

where $M^{A}$ is a scalar source. We emphasize that the term scalar in this section denotes an object, which does not carry matrix indices $(I J)$, although the object can be a vector, or a tensor in the color space. For example, the operator $V_{\gamma}^{a}$ in eq. (4.2) is a scalar, while the generator $t_{i j}^{a}$ is a matrix.

The operator $\phi$ has many common properties with the non-Abelian Wilson line $\Phi$. So, one may say that $\phi$ is a scalar image of the non-Abelian Wilson line. According to the discussion of section 3.1 the average of $\phi$ can be presented in the form of exponent

$$
\langle\phi\rangle=\frac{Z[M]}{Z[0]}=\exp W[M]
$$


The function $W[M]$ is given by all connected diagrams with insertions of operator $V_{A}$. The exponentiation of the scalar image of Wilson lines is, as simple as, the exponentiation of Wilson lines in the Abelian gauge theory, discussed in section 3.2. The only difference is that in the non-Abelian gauge theory the operator $V$ is given by infinite series of operators (4.6).

In order to fulfill the second point of our program, we need the formal definition of the matrix generalization procedure for a function of several arguments. It can be presented as action of the matrix shift operator on the scalar image,

$$
\widetilde{f}(T)_{i j}=\left.\left(e^{T^{A} \frac{\partial}{\partial x^{A}}}\right)_{i j} f(x)\right|_{x=0} .
$$

It is straightforward to check that right-hand-side of eq. (4.9) satisfies all standard demands on the matrix function $\widetilde{f}(T)$.

The Wilson line $\Phi$ is obtained from its scalar image (4.7) with the help of operation (4.9)

$$
\Phi_{I J}=\left.\left(e^{T^{A} \frac{\delta}{\delta M^{A}}}\right)_{I J} \phi\right|_{M=0} .
$$

Therefore, the average of Wilson line is given by

$$
\left\langle\Phi_{I J}\right\rangle=\left.\left(e^{T^{A} \frac{\delta}{\delta M^{A}}}\right)_{I J} \frac{Z[M]}{Z[0]}\right|_{M=0}=\frac{\widetilde{Z}_{I J}[T]}{Z[0]},
$$

where $\widetilde{Z}[T]$ is the matrix generalization of the partition function by means of the procedure (4.9).

As one can see from eq. (4.9), the generalization of the partition function to matrix sources is rather straightforward procedure. However, the relations between diagrams within $\widetilde{Z}[T]$ are not the same as within $Z[M]$. This happens due to the symmetrization of matrix variables by the shift operator (4.9). As a result, the originally disconnected diagrams are entangled by their matrix structure (see explicit example in eq. (4.24)). Therefore, the logarithm of the matrix partition function $\widetilde{Z}[T]$ is not the matrix generalization of the function $W[M]$ (which we denote as $\widetilde{W}[T]$ ). In symbolic notations this statement reads

$$
\frac{\widetilde{Z}_{I J}[T]}{Z[0]}=\left.\frac{1}{Z[0]}\left(e^{T^{A} \frac{\delta}{\delta M^{A}}}\right)_{I J} e^{W[M]}\right|_{M=0} \neq\left(e^{\widetilde{W}[T]}\right)_{I J}
$$

where

$$
\widetilde{W}_{I J}[T]=\left.\left(e^{T^{A} \frac{\delta}{\delta M^{A}}}\right)_{I J} W[M]\right|_{M=0} .
$$

The inequality (4.12) has been overlooked in ref. [9], therefore, the final result of ref. [9] presented there is incomplete.

Considering the left-hand-side of eq. (4.12) we conclude that although the function $\widetilde{W}$ is not the complete result of the exponentiation, but still it is the only function that can appear in the argument of the exponent. Taking into account that the leading term of the perturbative expansion is $\widetilde{W}$, we can present the left-hand-side of eq. (4.12) as

$$
\frac{\widetilde{Z}_{I J}[T]}{Z[0]}=\left(e^{\widetilde{W}[T]+\widetilde{\delta W}[T]}\right)_{I J},
$$

where $\widetilde{\delta W}$ is a function of $\widetilde{W}$. 
One can see that the function $\widetilde{W}$ plays an exceptional role in the GF exponentiation procedure. For this reason we call $\widetilde{W}$ the kernel of matrix exponentiation (MEK).

The correction terms $\widetilde{\delta W}$ we name the defect of matrix exponentiation (for shortness we often call it the defect). Mathematically, the defect arises from the reordering of colormatrices during the formation of matrix exponent. Accordingly, the structure of the defect resembles the famous tail of commutators in Baker-Campbell-Hausdorff $(\mathrm{BCH})$ formula. The defect is an algebraic function of $\widetilde{W}[T]$, in the same manner as the higher terms of $\mathrm{BCH}$ series are algebraic functions of the previous ones. The formal definition of the defect reads

$$
\begin{aligned}
\widetilde{\delta W}_{I J}[T] & =\left.\left[\ln ,\left(e^{T^{A} \frac{\delta}{\delta M^{A}}}\right)_{I J}\right] e^{W[M]}\right|_{M=0} \\
& =\ln \left(\left.\left(e^{T^{A} \frac{\delta}{\delta M^{A}}}\right)_{I J} e^{W[M]}\right|_{M=0}\right)-\left.\left(e^{T^{A} \frac{\delta}{\delta M^{A}}}\right)_{I J} \ln e^{W[M]}\right|_{M=0} .
\end{aligned}
$$

Thus, we have shown that the (T-)product of Wilson lines can be presented as a matrix exponent

$$
\left\langle\left(\Phi_{\gamma_{1}}^{f_{1}}\right)_{i_{1} j_{1}} \ldots\left(\Phi_{\gamma_{N}}^{f_{N}}\right)_{i_{N} j_{N}}\right\rangle=\left\langle\Phi_{I J}\right\rangle=\left(e^{\widetilde{W}[T]+\widetilde{\delta W}[T]}\right)_{I J}
$$

where functions $\widetilde{W}$ and $\widetilde{\delta W}$ are defined in (4.13) and (4.15) respectively. This expression is the main result of the article. In the following we discuss the properties of MEK and the defect, and demonstrate the relation between the presented approach and other approaches.

The form of the exponent (4.16) would necessarily appear during the exponentiation of any matrix object. The most important point of exponentiation is the selection of an efficient leading term, which is MEK in GF exponentiation. As we already told, in ref. [7] the whole perturbative series has been chosen as a leading term, and this choice leads to unnecessary complications. Our choice of MEK is inspired by many remarkable features of $\widetilde{W}$. Above all, MEK is the generating function for webs in the case of the scalar image of Wilson line. It implies that MEK already contains essential features of the exponentiated expression, except the matrix issues. Another example of important features favoring MEK is that MEK contains all color connected diagrams (see details in ref. [9]). The color connectivity is a defining attribute of web diagrams [8]. All in all, MEK is the best candidate for the leading term and, as we demonstrate later, such a conjecture is well-founded.

The structure presented in eq. (4.16) is can be also traced in the replica exponentiation $[5,6,8,10]$. Within the replica exponentiation the diagrams contributing to the exponent always has two contributions: the part proportional to unity in the replica space, and the rest, which has more involved structure (for explicit examples see e.g. equations (50), (61), (63) and (65) in [8]). The part proportional unity, after the summation over replica indices, is linear in $N_{\text {rep }}$, and therefore, directly contributes to the exponent. In GF exponentiation this part is given by MEK. The rest is an arbitrary polynomial of $N_{\text {rep }}$, the linear part of which contributes to the exponent. In GF exponentiation this contribution is given by the defect. We emphasize that in contrast to the replica exponentiation, 
in GF exponentiation the defect (4.15) is given by explicit expression, that significantly simplify the consideration of webs. The detailed comparison of the approaches, as well as, an example-calculation are given in section 5 .

\subsection{Structure of MEK}

MEK is a matrix generalization of the function $W[M]$ (4.8). Using the explicit expression for generating function (3.5) we obtain

$$
\widetilde{W}_{I J}[T]=T_{I J}^{A}\left\langle V^{A}\right\rangle+\frac{\left(T^{A} T^{B}\right)_{I J}}{2 !}\left\langle V^{A} V^{B}\right\rangle+\frac{\left(T^{A} T^{B} T^{C}\right)_{I J}}{3 !}\left\langle V^{A} V^{B} V^{C}\right\rangle+\ldots
$$

where we have used that the correlators are symmetric over permutations of operators.

Important to note that in the case of real gluon exchanges the matrix generalization of generating function is more involved. The product of T-ordered operators is not symmetric under permutations. Therefore, the expression (4.17) would contain terms with explicit symmetrization of generators.

Let us pass from the joined notation for multiple Wilson lines (4.3)-(4.4) to the consideration of individual contributions. We continue to assume that generators related to a separate Wilson line act in the separate matrix spaces. So, the generator $t_{a}^{\left[f_{k}\right]}$ is a matrix acting in the space $\left(i_{k}, j_{k}\right)$ and, MEK is a multimatrix in spaces $\left(i_{k}, j_{k}\right)$ for $k=1$ to $N$. For brevity we continue to denote the multi-index by capital letters, i.e. $W_{i_{1} \ldots i_{N}, j_{1} \ldots j_{N}}=W_{I J}$. We have

$$
T_{I J}^{A} V^{A}=\sum_{k=1}^{N}\left(t_{a}^{\left[f_{k}\right]}\right)_{i_{k} j_{k}} V_{\gamma_{k}}^{a},
$$

where the missed indices on the right-hand-side of the expression should be given by the unity matrices. Also we will omit the representation indication $[f]$ on generators, since it is fixed by denoting the matrix indices.

In the case of multiple Wilson lines, MEK reads

$$
\begin{aligned}
\widetilde{W}_{I J}= & \sum_{k=1}^{N} t_{i_{k} j_{k}}^{a}\left\langle V_{\gamma_{k}}^{a}\right\rangle+\sum_{\substack{k, l=1 \\
k \neq l}}^{N} \frac{t_{i_{k} j_{k}}^{a} t_{i_{l} j_{l}}^{b}}{2 !}\left\langle V_{\gamma_{k}}^{a} V_{\gamma_{l}}^{b}\right\rangle+\sum_{k=0}^{N} \frac{\left(t^{a} t^{b}\right)_{i_{k} j_{k}}}{2 !}\left\langle V_{\gamma_{k}}^{a} V_{\gamma_{k}}^{b}\right\rangle \\
& +\sum_{\substack{k, l, m=1 \\
k \neq l \neq m}}^{N} \frac{t_{i_{k} j_{k}}^{a} t_{i_{l} j_{l}}^{b} t_{i_{m} j_{m}}^{c}}{3 !}\left\langle V_{\gamma_{k}}^{a} V_{\gamma_{l}}^{b} V_{\gamma_{m}}^{c}\right\rangle+\sum_{\substack{k, l=1 \\
k \neq l}}^{N} \frac{\left(t^{a} t^{b}\right)_{i_{k} j_{k}} t_{i_{l} j_{l}}^{c}}{2 !}\left\langle V_{\gamma_{k}}^{a} V_{\gamma_{k}}^{b} V_{\gamma_{l}}^{c}\right\rangle \\
& +\sum_{k=1}^{N} \frac{\left(t^{a} t^{b} t^{c}\right)_{i_{k} j_{k}}}{3 !}\left\langle V_{\gamma_{k}}^{a} V_{\gamma_{k}}^{b} V_{\gamma_{k}}^{c}\right\rangle+\ldots .
\end{aligned}
$$


This can be simplified by the fixation of the order of individual Wilson line within correlator,

$$
\begin{aligned}
\widetilde{W}_{I J}= & \sum_{k=1}^{N} t_{i_{k} j_{k}}^{a}\left\langle V_{\gamma_{k}}^{a}\right\rangle+\sum_{\substack{k, l=1 \\
k<l}}^{N} t_{i_{k} j_{k}}^{a} t_{i_{l} j_{l}}^{b}\left\langle V_{\gamma_{k}}^{a} V_{\gamma_{l}}^{b}\right\rangle+\sum_{k=1}^{N} t_{\left.i_{k} j_{k}\right\}}^{\{a b\}}\left\langle V_{\gamma_{k}}^{a} V_{\gamma_{k}}^{b}\right\rangle \\
& +\sum_{\substack{k, l, m=1 \\
k<l<m}}^{N} t_{i_{k} j_{k}}^{a} t_{i_{l} j_{l}}^{b} t_{i_{m} j_{m}}^{c}\left\langle V_{\gamma_{k}}^{a} V_{\gamma_{l}}^{b} V_{\gamma_{m}}^{c}\right\rangle+\sum_{\substack{k, l=1 \\
k<l}}^{N} t_{i_{k} j_{k}}^{\left\{a b t_{i_{l} j_{l}}^{c}\right.}\left\langle V_{\gamma_{k}}^{a} V_{\gamma_{k}}^{b} V_{\gamma_{l}}^{c}\right\rangle \\
& +\sum_{\substack{k, l=1 \\
k<l}}^{N} t_{i_{k} j_{k}}^{a} t_{i_{l} j_{l}}^{\{b c\}}\left\langle V_{\gamma_{k}}^{a} V_{\gamma_{l}}^{b} V_{\gamma_{l}}^{c}\right\rangle+\sum_{k=1}^{N} t_{i_{k} j_{k}}^{\{a b c\}}\left\langle V_{\gamma_{k}}^{a} V_{\gamma_{k}}^{b} V_{\gamma_{k}}^{c}\right\rangle+\ldots,
\end{aligned}
$$

where $t^{\{a \ldots b\}}$ denotes the product of generators symmetrized over the indices

$$
t^{\left\{a_{1} \ldots a_{n}\right\}}=\frac{1}{n !} \sum_{\sigma=\operatorname{perm}\left[a_{1} \ldots a_{n}\right]} t^{\sigma_{1}} \ldots t^{\sigma_{n}} .
$$

According to eq. (4.20) one has a simple diagrammatic rule: MEK consists of all diagrams connecting arbitrary number of operators $V$ located on Wilson lines with unity symmetry coefficients. Every operator should be convoluted with the gauge group generator of the corresponded Wilson line. If there are several operators adjusted to the same Wilson line they should be convoluted with the symmetrized product of generators.

For the actual calculation one inserts the perturbative expansion for the operator $V$ in terms of operators (4.6). The operator $V_{n}$ radiates exactly $n$ gluons. Nonetheless, the general diagrammatic rules are still the same: the gluons could be contracted to other vertices, interact with each other, or be contracted to the same vertex, but all vertices $V$ must be connected together. An example of diagrams with three Wilson lines is demonstrated in figure 1.

\subsection{Structure of the defect}

The formal definition of the defect is given in eq. (4.15). However, the form (4.15) is not transparent and is hardy applicable. In this section we reorganize eq. (4.15) in simpler form, suitable for calculations.

In order to simplify eq. (4.15) we expand the exponent of the generating function in a series

$$
\begin{aligned}
e^{W[M]} & =1+W_{1}+\frac{W_{2}+W_{1}^{2}}{2}+\frac{W_{3}+3 W_{2} W_{1}+W_{1}^{3}}{3 !}+\ldots \\
& =\sum_{n=0}^{\infty} \sum_{\substack{\{k\} \\
\sum_{i} i \cdot k_{i}=n}} \frac{W_{1}^{k_{1}}}{(1 !)^{k_{1}} k_{1} !} \frac{W_{2}^{k_{2}}}{(2 !)^{k_{2}} k_{2} !} \cdots \frac{W_{n}^{k_{n}}}{(n !)^{k_{n}} k_{n} !},
\end{aligned}
$$

where we have introduced the short notation

$$
W_{k}=M^{A_{1}} \ldots M^{A_{k}}\left\langle V^{A_{1}} \ldots V^{A_{k}}\right\rangle .
$$




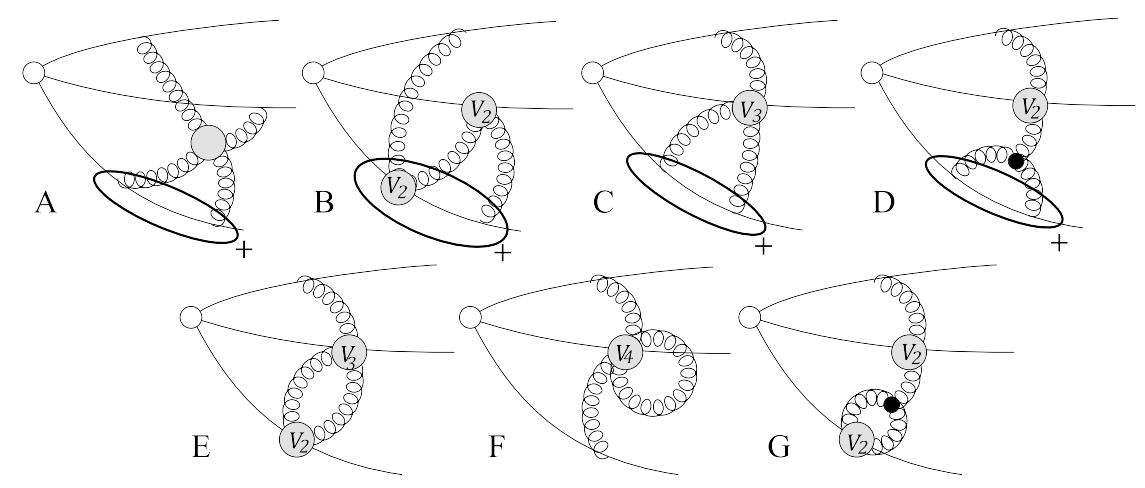

Figure 1. Diagrams contributing to MEK for three Wilson lines at $\mathcal{O}\left(g^{6}\right)$ order that connect all three Wilson lines (diagrams with permutations of Wilson lines should be added, as well as, web diagrams of $\mathcal{O}\left(g^{4}\right)$ order with a virtual loop). Blobs with $V_{n}$ denote the vertices (A.1), while the empty blob denotes all possible four-gluon tree interactions. Ovals with a "plus" sign denote the symmetrization of the vertices.

The action of the matrix shift exponent (4.9) replaces all sources $M^{A}$ by the generators $T^{A}$ in the completely symmetric way. This operation mixes the matrices, such that the result is not the matrix product of $\widetilde{W}_{n}$ 's. For example, the first term with mixture of matrices in eq. (4.22) is

$$
\left.\left(e^{T^{A} \frac{\delta}{\delta M^{A}}}\right)_{I J} W_{1} W_{2}\right|_{M=0}=\frac{1}{3}\left(T^{A} T^{B} T^{C}+T^{A} T^{C} T^{B}+T^{C} T^{A} T^{B}\right)_{I J}\left\langle V^{A} V^{B}\right\rangle\left\langle V^{C}\right\rangle
$$

where the symmetry of correlators is taken into account. One can see that this expression is not equivalent to $\left(\widetilde{W}_{1} \widetilde{W}_{2}+\widetilde{W}_{2} \widetilde{W}_{1}\right) / 2$, what is needed for the expansion of $\exp (\widetilde{W})$, but contains extra terms. The extra terms are proportional to commutators of generators and give rise to the defect of exponentiation.

Let us introduce the special notation for symmetrized products of the form (4.24). We denote

$$
\begin{aligned}
\left\{W_{n}\right\}_{I J} & =T_{I J}^{\left\{A_{1} \ldots A_{n}\right\}}\left\langle V^{A_{1}} \ldots V^{A_{n}}\right\rangle \\
\left\{W_{k} W_{n-k}\right\}_{I J} & =T_{I J}^{\left\{A_{1} \ldots A_{n}\right\}}\left\langle V^{A_{1}} \ldots V^{A_{k}}\right\rangle\left\langle V^{A_{k+1}} \ldots V^{A_{n}}\right\rangle \\
\left\{W_{k} W_{l} W_{n-k-l}\right\}_{I J} & =T_{I J}^{\left\{A_{1} \ldots A_{n}\right\}}\left\langle V^{A_{1}} \ldots V^{A_{k}}\right\rangle\left\langle V^{A_{k+1}} \ldots V^{A_{k+l}}\right\rangle\left\langle V^{A_{k+l+1}} \ldots V^{A_{n}}\right\rangle,
\end{aligned}
$$

etc.

where $T^{\{a \ldots b\}}$ denotes the symmetric product of generators, see (4.21).

The operation (4.25) does not have a special name, nonetheless it often appears in course of diagrammatic resummations and related problems. In ref. [7] this operation serves as a generalized product of diagrams needed for the diagrammatic exponentiation. Authors of ref. [7] shows that the result of the symmetrized product (4.25) has the meaning of the sum of all joined diagrams with all mutual ordering of gluons attached to Wilson lines. 
In the notation (4.25), the matrix generalization of the partition function reads

$$
\widetilde{Z}_{I J}[T]=\left.\left(e^{T^{A} \frac{\delta}{\delta M^{A}}}\right)_{I J} e^{W[M]}\right|_{M=0}=\sum_{n=0}^{\infty} \sum_{\substack{\{k\} \\ \sum_{i} i \cdot k_{i}=n}} \frac{\left\{W_{1}^{k_{1}} W_{2}^{k_{2}} \ldots W_{n}^{k_{n}}\right\}_{I J}}{(1 !)^{k_{1}}(2 !)^{k_{2}} \ldots(n !)^{k_{n}} k_{1} ! k_{2} ! \ldots k_{n} !} .
$$

In the same notation MEK and the partition function (4.26) are

$$
\widetilde{W}_{I J}[T]=\sum_{k=1}^{\infty} \frac{\left\{W_{k}\right\}_{I J}}{k !}, \quad \widetilde{Z}_{I J}[T]=\sum_{k=0}^{\infty} \frac{\left\{\widetilde{W}[T]^{k}\right\}_{I J}}{k !}
$$

where the operation $\{\ldots\}$ for $\left\{\widetilde{W}[T]^{k}\right\}$ should be taken individually for every term of perturbative expansion.

Finally, we evaluate (4.15) and obtain the expression for the defect. For the practical applications it is convenient to present the defect as the series

$$
\widetilde{\delta W}_{I J}[T]=\sum_{k=2}^{\infty}{\widetilde{\delta_{k} W}}_{I J}[T]
$$

where $\widetilde{\delta_{k} W} \sim \widetilde{W^{k}}$. The first few terms are

$$
\begin{aligned}
& {\widetilde{\delta_{2} W_{I J}}}_{I T]}=\frac{1}{2}\left(\left\{\widetilde{W}^{2}\right\}_{I J}-\left(\widetilde{W}^{2}\right)_{I J}\right) \text {, } \\
& {\widetilde{\delta_{3} W}}_{I J}[T]=\frac{1}{6}\left\{\widetilde{W}^{3}\right\}_{I J}-\frac{\left(\left\{\widetilde{W}^{2}\right\} \widetilde{W}\right)_{I J}+\left(\widetilde{W}\left\{\widetilde{W}^{2}\right\}\right)_{I J}}{4}+\frac{1}{3}\left(\widetilde{W}^{3}\right)_{I J}, \\
& \widetilde{\delta}_{4} W_{I J}[T]=\frac{\left\{\widetilde{W}^{4}\right\}_{I J}}{4 !}-\frac{\left(\left\{\widetilde{W}^{3}\right\} \widetilde{W}\right)_{I J}+\left(\widetilde{W}\left\{\widetilde{W}^{3}\right\}\right)_{I J}}{12}-\frac{\left(\left\{\widetilde{W}^{2}\right\}\left\{\widetilde{W}^{2}\right\}\right)_{I J}}{8} \\
& +\frac{\left(\left\{\widetilde{W}^{2}\right\} \widetilde{W}^{2}\right)_{I J}+\left(\widetilde{W}^{2}\left\{\widetilde{W}^{2}\right\}\right)_{I J}+\left(\widetilde{W}\left\{\widetilde{W}^{2}\right\} \widetilde{W}\right)_{I J}}{6}-\frac{\left(\widetilde{W}^{4}\right)_{I J}}{4} .
\end{aligned}
$$

The equations (4.29) can be significantly simplified by means of recursion. With this aim we consider eqs. (4.29) as equations on the totally symmetrized products $\left\{\widetilde{W}^{n}\right\}$, as functions of $\widetilde{\delta W}$. Substituting the solution again into eqs. (4.29) we found a simple recursive relation for the defect

$$
{\widetilde{\delta_{n} W}}_{I J}[T]=\frac{1}{n !}\left\{\widetilde{W}^{n}\right\}_{I J}-\sum_{k=2}^{n} \frac{1}{k !} \sum_{\substack{i>1 \\ \sum i=n}}\left(\widetilde{\delta_{i_{1} W}} \ldots \widetilde{\delta_{i_{k}} W}\right)_{I J},
$$

where $\widetilde{\delta_{1} W}=\widetilde{W}$. In the next sections we present explicit calculation of the defect for particular diagrams.

In fact, the matrix structure of the defect $\widetilde{\delta_{n} W}$ is proportional to (at least) $(n-1)$ commutators of generators. It follows from the recursive equation (4.30). Therefore, if matrices $T$ commute, all term of the defect are zero. In this way many contributions to the defect are zero, especially in for diagrams that involve many Wilson lines. 
Due to the property that the defect is proportional to the commutators of generators, the color factors appearing in the defect are color connected. Together with the statement that MEK is color connected, see discussion in [9], it proves the non-Abelian exponentiation theorem formulated in [8].

It is interesting to consider the gauge invariance of the exponentiated expression. Generally, the correlator (4.16) is not gauge invariant, and therefore, there are no special properties for MEK or for the defect. However, if one considers a color singlet configuration, or the open color indices located at spatial infinities, the correlator (4.16) is perturbative gauge invariant (for the later case, only in non-singular gauges, see e.g. discussion in ref. [15]). Consequently, the exponentiated expression for such a gauge-invariant configuration, is also gauge invariant. Indeed, at order $\alpha$ the statement is obvious. Given that, the gauge invariance for higher orders can be proven by iterations. In its own turn, the gauge invariance of the exponentiated expression leads to gauge invariance of the defect and MEK independently.

Let us sketch the proof of independent gauge invariance for MEK and the defect (given that the correlator under consideration is perturbative gauge invariant). The proof is made by interactions, comparing order-by-order the (gauge invariant) exponentiated expression and $\widetilde{W}[T]+\widetilde{\delta W}[T]$. Comparing the leading terms we observe that the leading term of MEK is gauge invariant. The next-to-leading term is given by the sum of next-to-leading term of MEK and leading term of the defect. The leading term of the defect is gauge invariant, because it is composed from the leading terms of MEK, see (4.30). Therefore, the next-to-leading term of MEK is gauge invariant. Then one can construct iteration and prove that MEK and the defect are independently gauge invariant at any order.

\section{Application of GF exponentiation}

In this section we compare the GF exponentiation with the diagrammatic and the replica exponentiations. The examples presented in the section clarify the role of the defect and MEK in the exponentiation procedure.

In order to compare approaches we consider two half-infinite Wilson lines, i.e. the cusp. The cusp is a popular playground for the testing of various methods. Recently, it has been evaluated up to the three loop order in the general kinematics in QCD [16]. The evaluation of exponentiated diagrams at one and two-loop order can be found in many articles, e.g. in refs. [17-20] one can find all necessary details and the explicit expressions. In the course of comparison we do not evaluate loop-integrals. Instead, we compare the unintegrated expressions for the diagrams and show the relations between different approaches.

In order to demonstrate the effectiveness of GF exponentiation we consider the special class of diagrams called the multiple gluon exchange webs (MGEW). These are the diagrams with neglected interaction between gluons. Such an approximation is simplest but non-trivial part of the exponentiated expression. Particulary, the simple structure of MGEWs results to the possibility to evaluate MGEW-loop-integrals at high orders without significant efforts, see e.g. [21, 22]. MGEWs were studied in details within the replica exponentiation in refs. [11, 22]. For simplicity, we consider the configuration of light-like Wilson 

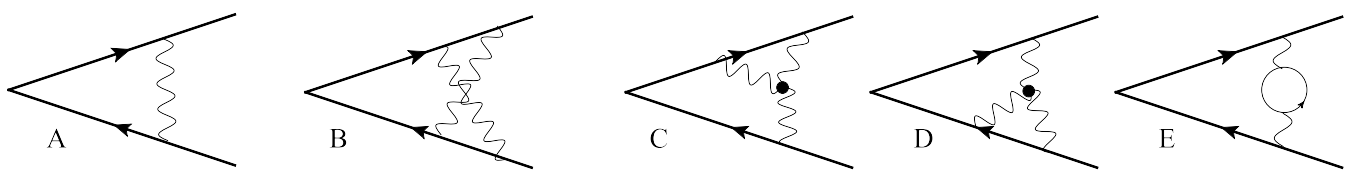

Figure 2. The web diagrams contributing to the correlator of two Wilson lines. The diagram B should be taken with the modified color factor.

lines, and show that in such kinematics MEK is given solely by one-loop diagrams. Therefore, all the higher order contribution are generated by the defect, and can be evaluated by algebraic manipulations.

\subsection{Cusp at two loops}

Let us consider a cusped Wilson line, which approaches from infinity to the origin along vector $v_{1}$, and then continues to infinity along vector $v_{2}$. For definiteness, we consider the Wilson line in the fundamental representation of $\mathrm{SU}\left(N_{c}\right)$. We denote

$$
\left\langle\left(\Phi_{v_{1}}^{\dagger} \Phi_{v_{2}}\right)_{i j}\right\rangle=\delta_{i j} \Gamma\left(v_{1}, v_{2}\right)=\delta_{i j} \exp \left[C_{F} E\left(v_{1}, v_{2}\right)\right]
$$

where $\Phi_{v}$ is the half-infinite Wilson line pointed from the origin along the vector $v$. For simplicity, we neglect self-interaction of Wilson lines, and use the Feynman gauge.

The webs contributing to $E$ within the diagrammatic exponentiation $[3,4]$ are presented on figure 2. The webs are to be equipped with modified color factors (2.2). At this order of accuracy, the only diagram, whose color factor differs from the original, is diagram B. Its color factor is $-C_{F} C_{A} / 2$.

In order to apply GF exponentiation as it is described in section 3, we consider both Wilson lines in separate matrix spaces, see section 4.1. At two-loop accuracy, only one combination of generators appears in the exponent. We denote

$$
\left\langle\left(\Phi_{v_{1}}^{\dagger}\right)_{i_{1} j_{1}}\left(\Phi_{v_{2}}\right)_{i_{2} j_{2}}\right\rangle=\exp \left[\left(t^{C}\right)_{i_{1} j_{1}}^{a} t_{i_{2} j_{2}}^{b} E^{a b}\left(v_{1}, v_{2}\right)+\ldots\right]
$$

where dots stay for other compositions of color indices arise at three-loop order (see example in the next section). Here, the exponent is the matrix exponent, where generators $t_{i_{1} j_{1}}$ and $t_{i_{2} j_{2}}$ act in different matrix spaces.

The label $C$ (as if charge-conjugation) on the first generator in eq. (5.2) recalls that these generators should be multiplied in the reverse order, from right to left. Such a prescription is needed to match out definition of the operator $V_{\gamma}$, which utilizes the pathordering from the origin, but not from infinity. In other words, the proper color and path ordering for conjugated Wilson line is $\Phi^{\dagger}=\exp \left(-t^{C} V\right)$, where the minus sign is the result of the conjugation of operator $V$.

Contracting eq. (5.2) with $\delta_{j_{1} i_{2}}$ and using that function $E^{a b}$ is proportional to $\delta^{a b}$, we obtain the relation (at this order of accuracy)

$$
E^{a b}\left(v_{1}, v_{2}\right)=\delta^{a b} E\left(v_{1}, v_{2}\right) .
$$

In its own turn, the function $E^{a b}$ is given by the sum of MEK and the defect (4.16). 


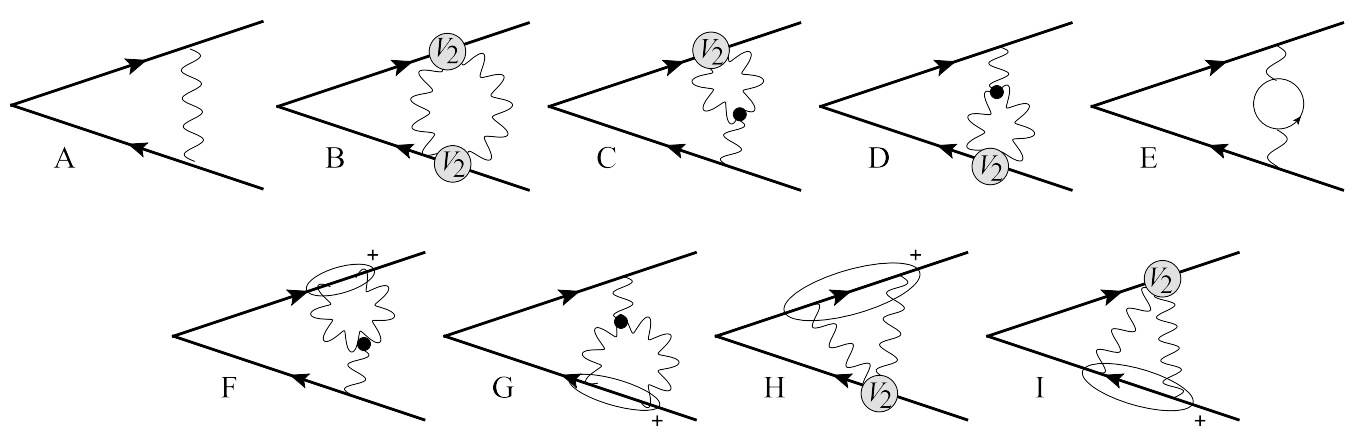

Figure 3. The diagrams contributing to MEK for the cusp. The diagram B does not equal the diagram B in figure 2. Diagrams C, D and E, as well as, the diagram A are equal to the corresponding diagrams in figure 2. Diagrams F, G, H and I are zero due to the convolution of antisymmetric three-gluon vertex with the symmetrized product of generators.

Let us remark, that one can consider the cusped Wilson line in another way. Instead of consideration of every Wilson line in separate color space, as it is done in (5.2), one can consider a single Wilson line, whose path-dependent part is split, $V_{\gamma}^{a}=V_{v_{1}}^{a}+V_{v_{2}}^{a}$. Within such consideration, one can keep a single generator $t^{a}$ in (4.2) by the price of two operators $V$. These two approaches are equivalent to each other, although diagrams would distribute differently within the perturbative expansion of MEK (4.20).

In the following we perform the diagram-by-diagram comparison for both sides of (5.3). The diagrams contributing to MEK are presented in figure 3. In the context of eq. (4.20), the diagrams from $\mathrm{A}$ to $\mathrm{E}$ are produced by the perturbative expansion of the double correlator, while the diagrams $\mathrm{F}$ and $\mathrm{G}$ are produced by the triple correlator. Contribution of the average of an operator $V$ is zero in the absence of self-interaction.

Let us compare diagrams one-by-one. The comparison is simpler to perform in the position representation. The gluon propagator reads

$$
\Delta_{a b}^{\mu \nu}(x, y)=g^{\mu \nu} \delta_{a b} \Delta(x, y)=\frac{\Gamma(1-\epsilon)}{4 \pi^{2-\epsilon}} \frac{-g^{\mu \nu} \delta_{a b}}{\left(-(x-y)^{2}+i 0\right)^{1-\epsilon}}
$$

where $\epsilon$ is the parameter of the dimension regularization, $d=4-2 \epsilon$. The expressions for the vertices $V_{n}$ are presented in appendix A.

The diagrams $\mathrm{A}$ and $\mathrm{E}$ stay the same in both approaches. For the following consideration we need the one-loop expression for MEK,

$$
\widetilde{W}_{1 \text {-loop }}=\left(t^{C}\right)_{i_{1} j_{1}}^{a} t_{i_{2} j_{2}}^{b} W_{1-\text { loop }}^{a b}=-(i g)^{2}\left(t^{C}\right)_{i_{1} j_{1}}^{a} t_{i_{2} j_{2}}^{a} v_{12} \int_{0}^{\infty} d x d y \Delta\left(v_{1} x, v_{2} y\right),
$$

where $v_{i j}=\left(v_{i} \cdot v_{j}\right)$.

The diagrams $\mathrm{C}$ and $\mathrm{D}$ are also equal in both approaches. Let us demonstrate it explicitly. The diagram $\mathrm{C}$ in figure 2 reads

$$
\delta_{i j} C_{F} E_{C}=(i g)^{3}\left(t^{a} t^{b} t^{c}\right)_{i j} i f^{a b c} \mathcal{F}_{C}=\delta_{i j} C_{F} \frac{i C_{A}}{2} \mathcal{F}_{C}
$$


The kinematical part equals to

$$
\mathcal{F}_{C}=\int_{0}^{\infty} d y d x_{1,2} \int d^{d} z V_{\mu \nu \rho}(z) v_{1}^{\mu} v_{1}^{\nu} v_{2}^{\rho} \Delta\left(z, v_{1} x_{1}\right) \Delta\left(z, v_{1} x_{2}\right) \Delta\left(z, v_{2} y\right) \theta\left(x_{2}<x_{1}\right),
$$

where $V^{\mu \nu \rho}(z)$ is the Feynman rule for the triple-gluon vertex. The diagram $\mathrm{C}$ in figure 3 reads

$$
E_{C}^{a b}=\frac{1}{2} \frac{(i g)\left(i g^{2}\right)}{2}\left(f^{a d c}-f^{a c d}\right) i f^{c d b} \mathcal{F}_{C}=\delta^{a b} \frac{i C_{A}}{2} \mathcal{F}_{C}
$$

where we have used the Feynman rules for $V_{2}$ (A.4) and the symmetry properties of the three-gluon vertex. The factor $1 / 2$ in front of expression (5.7) is the symmetry coefficient of the diagram. Therefore, we find that $E_{C}^{a b}=\delta^{a b} E_{C}$. The consideration of the diagram D is analogous.

The diagrams F, G, H and I have not analogues in the diagrammatic exponentiation. These diagrams equal zero, due to the convolution of the symmetric combination of generators with the anti-symmetric color structure of the three-gluon vertex, or $V_{2}$ vertex. Explicitly, we have

$$
\left(t^{C}\right)_{i_{1} j_{1}}^{a} t_{i_{2} j_{2}}^{b} E_{F}^{a b}\left(v_{1}, v_{2}\right)=(i g)^{3}\left(t^{\{a b\}}\right)_{i_{1} j_{1}} t_{i_{2} j_{2}}^{c} i f^{a b c} \mathcal{F}_{F}=0
$$

where the kinematical part is

$$
\mathcal{F}_{F}=\int_{0}^{\infty} d y d x_{1,2} \int d^{d} z V_{\mu \nu \rho}(z) v_{1}^{\mu} v_{1}^{\nu} v_{2}^{\rho} \Delta\left(z, v_{1} x_{1}\right) \Delta\left(z, v_{1} x_{2}\right) \Delta\left(z, v_{2} y\right) .
$$

The consideration of diagrams $\mathrm{G}, \mathrm{H}$ and $\mathrm{I}$ is analogous.

Finally, the diagrams B in figure 2 and in figure 3 are not equal. This is the manifestation of the matrix origin of Wilson lines. Within the diagrammatic exponentiation the diagram $B$ reads

$$
E_{B}=-(i g)^{4} \frac{C_{A}}{2} v_{12}^{2} \times \int_{0}^{\infty} d x_{1,2} d y_{1,2} \Delta\left(v_{1} x_{1}, v_{2} y_{1}\right) \Delta\left(v_{1} x_{2}, v_{2} y_{2}\right) \theta\left(x_{1}<x_{2}\right) \theta\left(y_{2}<y_{1}\right) .
$$

While, in GF exponentiation the contribution of the diagram B to MEK reads

$$
\begin{aligned}
E_{B, \mathrm{MEK}}^{a b}= & \frac{-1}{2}\left(\frac{i g^{2}}{2}\right)^{2} f^{a c d} f^{b c d} v_{12}^{2} \int_{0}^{\infty} d x_{1,2} d y_{1,2} \\
& \times \Delta\left(x_{1}, y_{1}\right) \Delta\left(x_{2}, y_{2}\right)\left(\theta\left(x_{1}<x_{2}\right)-\theta\left(x_{2}<x_{1}\right)\right)\left(\theta\left(y_{1}<y_{2}\right)-\theta\left(y_{2}<y_{1}\right)\right) .
\end{aligned}
$$

The factor $1 / 2$ in front of the expression is the symmetry coefficient.

Expressions (5.9) and (5.10) differ significantly. The difference is the defect of exponentiation, which comes from the squaring of the one-loop contribution (5.5). Evaluating the expression (4.29) we obtain

$$
\begin{aligned}
\widetilde{\delta_{2} W}= & \frac{1}{2}\left[\left(t^{\left\{a_{1} a_{2}\right\}}\right)_{i_{1} j_{1}}^{C} t_{i_{2} j_{2}}^{\left\{b_{1} b_{2}\right\}}-\left(t^{a_{2}} t^{a_{1}}\right)_{i_{1} j_{1}}^{C}\left(t^{b_{1}} t^{b_{2}}\right)_{i_{2} j_{2}}\right] W_{1-\text { loop }}^{a_{1} b_{1}} W_{1-\text { loop }}^{a_{2} b_{2}} \\
= & \frac{(i g)^{4}}{2}\left[\frac{\left(t^{a} t^{b}+t^{b} t^{a}\right)_{i_{1} j_{1}}^{C}}{2} \frac{\left(t^{a} t^{b}+t^{b} t^{a}\right)_{i_{2} j_{2}}}{2}-\left(t^{b} t^{a}\right)_{i_{1} j_{1}}^{C}\left(t^{a} t^{b}\right)_{i_{2} j_{2}}\right] v_{12}^{2} \\
& \times \int_{0}^{\infty} d x_{1,2} d y_{1,2} \Delta\left(v_{1} x_{1}, v_{2} y_{1}\right) \Delta\left(v_{1} x_{2}, v_{2} y_{2}\right) .
\end{aligned}
$$


Simplifying the color structure we find

$$
E_{B, \text { defect }}^{a b}=\frac{\left(i g^{2}\right)^{2}}{8} f^{a c d} f^{b c d} v_{12}^{2} \int_{0}^{\infty} d x_{1,2} d y_{1,2} \Delta\left(v_{1} x_{1}, v_{2} y_{1}\right) \Delta\left(v_{1} x_{2}, v_{2} y_{2}\right) .
$$

Adding the defect contribution (5.12) to MEK contribution (5.10) and simplifying the integrands we obtain

$$
E_{B, \mathrm{MEK}}^{a b}+E_{B, \text { defect }}^{a b}=\delta^{a b} E_{B} .
$$

Therefore, the sum of diagram B for MEK and the defect reproduces the diagram B within the diagrammatic exponentiation.

Thus, we observe the complete agreement between GF exponentiation and the diagrammatic exponentiation. We stress that the correspondence between approaches is not trivial. Namely, the diagrams contributing to MEK have an unique kinematical part. Only in the combination with the defect, the standard diagrammatic (2.1) is restored. It implies that GF exponentiation exponentiates not only the color part but also the kinematic part. So, the method separates unique contributions of two-loop integrals, from the powers of one-loop contribution.

It is also instructive to compare GF exponentiation with the replica exponentiation. The diagrams appearing in replicated theory are the same as shown in figure 3 . The expressions to compare in the form of eq. (5.2) can be found in ref. [8].

The contributions of diagrams A, C, D, E, F and G equal in both approaches. The equivalence happens because these diagrams are irreducible in the absence of Wilson lines. Therefore, only the fields of the same replica quantum number are presented in the diagram. In other words, these diagrams are proportional to unity in the replica space, that after evaluation of the diagrams results in the common factor $N_{\text {rep }}$ (number of replicas). Effective vertices at $N_{\text {rep }}=1$ are the same in both approaches (compare our definition of $V$ in (4.2) with the definition (20) in ref. [8]. Also compare explicit expressions for the vertices $V_{n}$ (A.1) with (22a)-(22c) in ref. [8]). Thus, the rest parts of diagrams are identically equal in both approaches.

Diagrams $\mathrm{H}$ and I are zero in both approaches due to the convolution of symmetric and anti-symmetric color factors. In ref. [8] this fact is demonstrated in eq. (48).

Diagram B in the replica exponentiation is presented in eq. (52) of ref. [8]. Using our notation this equation reads

$$
\begin{aligned}
E_{B, \text { rep }}^{a b}= & -\frac{\left(i g^{2}\right)^{2}}{8} f^{c d a} f^{c d b} v_{12}^{2} \int_{0}^{\infty} d x_{1,2} d y_{1,2} \Delta\left(v_{1} x_{1}, v_{2} y_{1}\right) \Delta\left(v_{1} x_{2}, v_{2} y_{2}\right) \\
& \times\left[N_{\text {rep }}\left(\theta\left(x_{1}<x_{2}\right)-\theta\left(x_{2}<x_{1}\right)\right)\left(\theta\left(y_{1}<y_{2}\right)-\theta\left(y_{2}<y_{1}\right)\right)+N_{\text {rep }}\left(N_{\text {rep }}-1\right)\right],
\end{aligned}
$$

where we have taken into account that in ref. [8] both Wilson lines are incoming. Comparing this expression with (5.10) and (5.12) we observe that

$$
E_{B, \text { rep }}^{a b}=N_{\text {rep }} E_{B, \text { MEK }}^{a b}-N_{\text {rep }}\left(N_{\text {rep }}-1\right) E_{B, \text { defect }}^{a b} .
$$

Therefore, the contribution of MEK and the defect are clearly distinguished within the replica exponentiation. Namely, the diagrams with only a single copy of field (i.e. with 


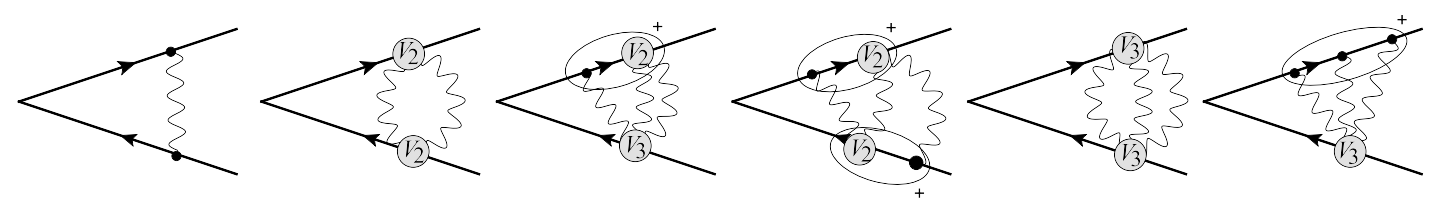

Figure 4. MGEWs contributing to the cusp at three-loop level order. The symmetric diagrams are not shown. For light-like Wilson lines only the first diagram is not zero.

all internal vertices proportional to $\delta_{n m}$ in replica space) are the contribution to MEK. These diagrams are proportional to the first power of $N_{\text {rep }}$. The rest contributions are proportional to a polynomial in $N_{\text {rep }}$ and represent the contribution of the defect. The polynomial in $N_{\text {rec }}$ reflects the reordering of color matrices during exponentiation, which is taken into account explicitly by definition (4.15) in the case of GF exponentiation.

\subsection{Multiple gluon exchange webs for light-like Wilson lines}

The webs, as well as, diagrams contributing to MEK are naturally split on subclasses by the number of connected parts of the internal graph. The division by this principle is not gauge invariant, but it is very effective from the practical point of view.

The limiting case of such a division is the completely connected diagrams, i.e. diagrams that are connected in the absence of both Wilson lines and vertices $V_{n}$ (e.g. these are diagrams A, C, D, E, F, G in figure 3, and diagram A in figure 1). These diagrams are not influenced by the matrix structure of the non-Abelian gauge theory, and do not mix with the defect. For this subclass, the diagrams of MEK are in one-to-one correspondence with the diagrams of the diagrammatic exponentiation. The limit of completely connected graphs resembles the Abelian exponentiation procedure.

The opposite limiting case of the division is the diagrams without any internal interaction of gluons (e.g. these are diagrams B,C,E, and F in figure 1). These diagrams have been studied in details in the recent publications [11,22], and are called multiple gluon exchange webs (MGEW). Within GF exponentiation MGEWs are given by the sum of MEK and the defect contributions. Moreover, the contribution of the defect is the strongest in comparison to the more connected diagrams. In some sense, MGEWs contain the smallest amount of a new (in comparison to the previous order of perturbative calculation) information.

MGEWs are the best demonstration of the effectiveness of GF exponentiation. In the case of light-like Wilson lines MEK can be evaluated exactly, and directly contributes only at one-loop order. Therefore, for light-like Wilson lines the higher-loop orders of MGEWs are given entirely by the defect of exponentiation.

To start with, we consider the cusp configuration (5.2) at $v_{1}^{2}=v_{2}^{2}=0$. MGEWs contributing to the cusp up to three-loop level order are shown in figure 4 . The one-loop contribution to MEK is given by (5.5). For the light-like kinematic we have

$$
E_{A}^{a b}=-\delta^{a b} g^{2} v_{12}^{\epsilon} \frac{\Gamma(1-\epsilon)}{4 \pi^{2-\epsilon}} \mu^{2 \epsilon} \int_{0}^{\infty} \frac{d x d y}{(2 x y+i 0)^{1-\epsilon}} e^{-\delta(x+y)}
$$


where exponent performs the infrared regularization. For future convenience we rewrite the one-loop expression in the form

$$
E_{A}^{a b}=-\delta^{a b} \alpha_{s} v_{12}^{\epsilon} K_{1}, \quad K_{1}=\frac{\Gamma^{2}(\epsilon) \Gamma(1-\epsilon)}{(2 \pi)^{1-\epsilon}}\left(\frac{\mu^{2}}{\delta^{2}}\right)^{\epsilon} .
$$

The two-loop contribution to MEK is given in eq. (5.10). In the light-like kinematics it reads

$$
\begin{aligned}
E_{B, \mathrm{MEK}}^{a b}= & \frac{-1}{2}\left(\frac{i g^{2}}{2}\right)^{2} f^{a c d} f^{b c d} v_{12}^{2 \epsilon} \frac{\Gamma^{2}(1-\epsilon)}{16 \pi^{4-2 \epsilon}} \int_{0}^{\infty} d x_{1,2} d y_{1,2} \\
& \times \frac{\left(\theta\left(x_{1}<x_{2}\right)-\theta\left(x_{2}<x_{1}\right)\right)\left(\theta\left(y_{1}<y_{2}\right)-\theta\left(y_{2}<y_{1}\right)\right)}{\left(2 x_{1} y_{1}+i 0\right)^{1-\epsilon}\left(2 x_{2} y_{2}+i 0\right)^{1-\epsilon}} e^{-\delta\left(x_{1}+x_{2}+y_{1}+y_{2}\right)} .
\end{aligned}
$$

The $i 0$ prescription does not influence the integral, because the ultraviolet singularity at $x y \rightarrow 0$ is regulated by the dimension regularization. We conclude that the propagator attached to light-like Wilson lines can be split on individual components, $\Delta \sim x^{\epsilon-1} y^{\epsilon-1}$. Therefore, the loop integral is zero due to the antisymmetry of the integrand under the permutation of $x_{1}$ and $x_{2}$, or $y_{1}$ and $y_{2}$.

Thus, in the light-like kinematics of Wilson line at two-loop MGEW contribution is given solely by the defect contribution. Evaluating integral (5.12) we obtain

$$
E_{2 \text {-loop,MGEW }}^{a b}=-\alpha_{s}^{2} \frac{C_{A}}{8} v_{12}^{2 \epsilon} K_{1}^{2} .
$$

This result coincides with the calculation performed in the diagrammatic exponentiation, see e.g. [20].

In the similar way one can show that the contribution of the three- and higher-loop diagrams to MEK are zero. Indeed, all the propagators for MGEW with light-like Wilson lines are just the product of components, $\Delta \sim x^{\epsilon-1} y^{\epsilon-1}$. Therefore, loop-integrals can be split on two parts: one depending on the variables related to Wilson line $\Phi_{v_{1}}$, and another depending on the variables related to $\Phi_{v_{2}}$. The interaction with Wilson lines are given by vertices $V_{n}$. Schematically, the contribution of some n-loop diagram reads

$$
\sum \text { (color factor) } \int_{0}^{\infty} d x_{1, \ldots, n} \frac{V_{k}\left(x_{1}, \ldots\right) \ldots V_{l}\left(\ldots, x_{n}\right)}{x_{1}^{1-\epsilon} \ldots x_{n}^{1-\epsilon}} \int_{0}^{\infty} d y_{1, \ldots, n} \frac{V_{r}\left(y_{1}, \ldots\right) \ldots V_{s}\left(\ldots, y_{n}\right)}{y_{1}^{1-\epsilon} \ldots y_{n}^{1-\epsilon}}
$$

where the sets of vertices $(k, \ldots, l)$ and $(r, \ldots, s)$ are defined by the diagram, $k+\ldots+l=$ $r+\ldots+l=n$, and the summation runs over all independent color structures. According to the diagrammatic rules for MEK, there are necessarily several vertices $V_{n}$ with $n>1$. The kinematical part of vertices $V_{n}\left(x_{1}, \ldots, x_{n}\right)$ is antisymmetric over the permutation of arguments. Therefore, the integrals on the right-hand-side of (5.20) are zero. The only exception is the case when all vertices are $V_{1}$, which is the one-loop contribution (5.17).

We conclude that in the MGEW approximation, MEK is given solely by the one-loop diagram, and reads

$$
\left.\widetilde{W}_{I J}\left(v_{1}, v_{2}\right)\right|_{\mathrm{MGEW}}=-\left(t^{C}\right)_{i_{1} j_{1}}^{a} t_{i_{2} j_{2}}^{a} \alpha_{s} v_{12}^{\epsilon} K_{1}
$$

where $K_{1}$ is given in (5.17). 


\begin{tabular}{|l|l|l|l|}
\hline$n$ & $E_{n, t t}$ & $E_{n, \delta \delta}$ & $E_{n, \text { cusp }}$ \\
\hline 1 & -1 & 0 & -1 \\
\hline 2 & $-\frac{N_{c}}{8}$ & 0 & $-\frac{N_{c}}{8}$ \\
\hline 3 & $\frac{3-2 N_{c}^{2}}{72}$ & $\frac{1-N_{c}^{2}}{48}$ & $-\frac{N_{c}^{2}}{36}$ \\
\hline 4 & $\frac{N_{c}\left(40-33 N_{c}^{2}\right)}{4608}$ & $\frac{5 N_{c}\left(1-N_{c}^{2}\right)}{768}$ & $\frac{N_{c}\left(10-33 N_{c}^{2}\right)}{4608}$ \\
\hline 5 & $\frac{-35+93 N_{c}^{2}-57 N_{c}^{4}}{28800}$ & $\frac{\left(1-N_{c}^{2}\right)\left(23 N^{2}-22\right)}{23040}$ & $\frac{N_{c}^{2}\left(71-114 N_{c}^{2}\right)}{57600}$ \\
\hline
\end{tabular}

Table 1. The coefficients for expansion of the light-like cusp amplitude in MGEW approximation (5.22)-(5.23) for $\mathrm{SU}\left(N_{c}\right)$ gauge theory.

The fact that at MGEW approximation MEK is given by solely by one-loop diagram, leads to the surprising conclusion. Namely, MGEW approximation is gauge invariant (in non-singular gauges) in the case of light-like kinematics, as long as, Wilson lines are color connected at the origin. Indeed, MEK is given by a single gauge invariant diagram. As a consequence, the defect is also gauge invariant, see discussion in the end of section 4.4. The gauge invariance of MGEW approximation holds only for light-like Wilson lines. For the Wilson lines off-light-cone the MEK has contributions of higher orders, which are composed from different diagrams, and therefore, MGEW diagrams are not gauge invariant.

The contribution of the defect can be obtained by straightforward evaluation of expressions (4.29) with MEK (5.21). For an arbitrary gauge group the evaluation involves the elaboration of higher Casimir operators (see e.g. four-loop example in [21]). However, for any given algebra the evaluation can be performed easily. It is convenient to present the final result for two Wilson lines in the form

$$
\left.\left\langle\left(\Phi_{v_{1}}^{\dagger}\right)_{i_{1} j_{1}}\left(\Phi_{v_{2}}\right)_{i_{2} j_{2}}\right\rangle\right|_{\mathrm{MGEW}}=\exp \left[\sum_{n=1}^{\infty} \alpha_{s}^{n} v_{12}^{n \epsilon} K_{1}^{n}\left(\left(t^{C}\right)_{i_{1} j_{1}}^{a} t_{i_{2} j_{2}}^{a} E_{n, t t}+\frac{\delta_{i_{1} j_{1}} \delta_{i_{2} j_{2}}}{N_{c}} E_{n, \delta \delta}\right)\right],
$$

where $E_{n}$ 's are numeric coefficients. Here, the exponent is the matrix exponent, where matrices with indices $\left(i_{1} j_{1}\right)$ and $\left(i_{2} j_{2}\right)$ belongs to different matrix spaces. Often one considers the cusp configuration with contracted color indices. Then it is convenient to use the following parametrization

$$
\left.\left\langle\left(\Phi_{v_{1}}^{\dagger} \Phi_{v_{2}}\right)_{i j}\right\rangle\right|_{\mathrm{MGEW}}=\delta_{i j} \exp \left[C_{F} \sum_{n=1}^{\infty} \alpha_{s}^{n} v_{12}^{n \epsilon} K_{1}^{n} E_{n, \text { cusp }}\right] .
$$

The first few coefficients $E$ for the case of $\mathrm{SU}\left(N_{c}\right)$ gauge group are presented in table 1 . For the calculation we have used the ColorMath package [23].

In the case of many Wilson lines the general consideration remains the same. Since the diagrams contributing to MEK are connected in the absence of Wilson lines they necessarily contain several vertices $V_{n}$ with $n>1$, see the example of two-loop MGEWs with four Wilson lines in figure 5, and three-loop MGEWs with three Wilson lines in 


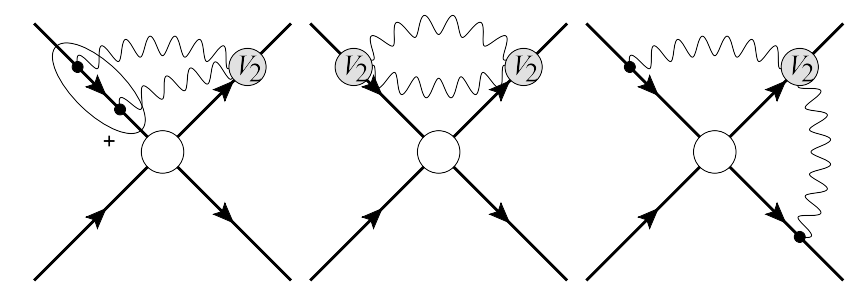

Figure 5. MGEW contributing to MEK for the correlator of four Wilson lines. The diagrams with permuted Wilson lines should be added. For light-like Wilson lines these diagrams are zero.

figure 1 (the diagrams B,C,E and F). The only non-zero contribution to MEK is given by the one-loop diagrams. Therefore, MEK is given by

$$
\left.\widetilde{W}_{I J}\left(v_{1}, \ldots, v_{n}\right)\right|_{\mathrm{MGEW}}=\sum_{\substack{k, l=1 \\ k<l}}^{n} t_{i_{k} j_{k}}^{a} t_{i_{l} j_{l}}^{a} \alpha_{s} v_{k l}^{\epsilon} K_{1},
$$

where $K_{1}$ is given in (5.17), and missed indices should be saturated by Kronecker deltas. The expression (5.24) is given for the Wilson lines pointing from the origin. In the case of Wilson line incoming to the origin the corresponded generator should be replaced by $-t^{C}$. Thus, the only non-trivial contribution to the correlator of light-like Wilson lines in MGEW approximation comes from the defect and can be evaluated algebraically.

As an illustrative example, we present the correlator of four light-like Wilson lines in MGEW approximation in the exponentiated form. The complete result (with eight open indices) is rather involved. Therefore, we took a particular convolution of Wilson lines, which corresponds to the scattering in $t$-channel. In MGEW approximation such a correlator is convenient to present in the following form

$$
\left.\left\langle\left(\Phi_{v_{1}}^{\dagger} \Phi_{v_{3}}\right)_{i_{1} j_{1}}\left(\Phi_{v_{2}}^{\dagger} \Phi_{v_{4}}\right)_{i_{2} j_{2}}\right\rangle\right|_{\mathrm{MGEW}}=\exp \left(-2 \sum_{n=1}^{\infty} \alpha_{s}^{n} K_{1}^{n}\left[\delta_{i_{1} j_{1}} \delta_{i_{2} j_{2}} A_{n}+\delta_{i_{1} j_{2}} \delta_{i_{2} j_{1}} B_{n}\right]\right) .
$$

The expression for coefficients $A$ and $B$ are presented in table 2 up to the three-loop order. The variables $s, t$ and $u$ are defined as

$$
\begin{aligned}
& s_{n}=\left(v_{12}\right)^{n \epsilon}+\left(v_{34}\right)^{n \epsilon}, \quad t_{n}=\left(v_{14}\right)^{n \epsilon}+\left(v_{23}\right)^{n \epsilon} \quad u_{n}=\left(v_{13}\right)^{n \epsilon}+\left(v_{24}\right)^{n \epsilon} \\
& \theta_{1}=\left(v_{12} v_{13} v_{23}\right)^{\epsilon}+\left(v_{12} v_{14} v_{24}\right)^{\epsilon}+\left(v_{13} v_{14} v_{34}\right)^{\epsilon}+\left(v_{23} v_{24} v_{34}\right)^{\epsilon} .
\end{aligned}
$$

The calculation of higher orders in MGEW approximation does not present any difficulties, but also is not interesting. We remind that MGEW is only an approximation, and therefore, it can be considered only as an illustration. Nonetheless, the results presented in table 2 show some interesting features.

One can see that the two-loop expression resembles the one-loop expression. This is a known feature of the two-loop soft anomalous dimension, see [24, 25]. Moreover the proportionality coefficient is the same as in MGEW approximation for the cusp, see $E_{(1,2), \text { cusp }}$ in table 1 . In fact, it is a consequence of a general statement: in two-loop 


\begin{tabular}{|c|c|}
\hline$n$ & $A_{n}, B_{n}$ \\
\hline 1 & $A_{1}=2 C_{F} u_{1}+\frac{s_{1}-t_{1}}{N_{c}}$ \\
\hline 2 & $A_{2}=\frac{C_{A}}{8}\left(2 C_{F} u_{2}+\frac{s_{2}-t_{2}}{N_{c}}\right)$ \\
\hline 3 & $\begin{aligned} A_{3}= & \frac{C_{A}^{2}}{36}\left[2 C_{F} u_{3}+\frac{3}{4 N_{c}}\left(\frac{7 s_{3}}{3}+\frac{2 t_{3}}{3}+\frac{s_{2}\left(3 u_{1}-5 t_{1}\right)}{2}\right.\right. \\
& \left.\left.-2 t_{2} s_{1}+u_{2}\left(s_{1}-t_{1}\right)+s_{1}^{2}\left(t_{1}-u_{1}\right)+\frac{t_{1} u_{1}^{2}-t_{1}^{2} u_{1}+s_{1}\left(t_{1}^{2}-u_{1}^{2}\right)}{2}+\frac{3 \theta_{1}}{2}\right)\right] \\
B_{3}= & \frac{C_{A}^{2}}{144}\left[C_{F}\left(8 t_{3}-2 s_{3}-2 s_{2}\left(t_{1}+u_{1}\right)+6 t_{2}\left(u_{1}-s_{1}\right)+6 u_{2}\left(t_{1}-s_{1}\right)\right.\right. \\
& \left.+3 s_{1}\left(t_{1}^{2}+u_{1}^{2}\right)-3 t_{1} u_{1}\left(t_{1}+u_{1}\right)+3 \theta_{1}\right) \\
& +\frac{3}{N_{c}}\left(-\frac{7 s_{3}}{3}-\frac{2 t_{3}}{3}+2 s_{1} t_{2}+\frac{s_{2}}{2}\left(5 t_{1}-3 u_{1}\right)+u_{2}\left(t_{1}-s_{1}\right)\right. \\
& \left.\left.+\left(t_{1}-u_{1}\right)\left(\frac{u_{1} t_{1}}{2}-s_{1}^{2}\right)+\frac{s_{1}^{2}}{2}\left(u_{1}^{2}-t_{1}^{2}\right)-\frac{3 \theta_{1}}{2}\right)\right]\end{aligned}$ \\
\hline
\end{tabular}

Table 2. The coefficients for expansion of correlator of four light-like Wilson lines in MGEW approximation (5.25). The variables $s, t, u$ and $\theta_{1}$ are defined in (5.26).

approximation the soft anomalous dimension (for light-like Wilson lines) is equal to the oneloop anomalous dimension multiplied by the two-loop cusp anomalous dimension [24, 25]. This observation is known as the dipole formula for infrared singularities of scattering amplitudes in QCD [26, 27]. The status of the dipole formula at three-loop order and higher is currently questionable, e.g. see ref. [28], for recent review see ref. [29].

Considering, the expression presented in table 2, we observe that at three-loop order MGEW approximation does not support the dipole formula. Since in the considered kinematics MGEW approximation is gauge invariant, the expression given in tables 1 and 2 represent some irreducible part of the complete result. In this way we conclude that the violation of the dipole formula at the three-loop order is likely.

\section{Conclusion}

In this article we have presented an approach that allows one to evaluate the correlator of a product of Wilson lines directly in the exponentiated form. In contrast to the existing methods of exponentiation, e.g. [3-7], the presented method does not rely on the diagrammatical consideration, and allows to obtain the argument of exponent in the form of correlators of known operators. To our best knowledge, it is the first example for such a relation in a non-Abelian gauge theory.

The approach is based on the fundamental property of the perturbative expansion, namely, on the exponentiation of the connected part of Feynman diagrams. In section 3.1 (see also [9]) we show that this property implies the exponentiation of the diagrammatic expansion for a wide class of operators; the operators, which can be presented in the form 
of the exponent (3.3). Although this observation is evident and well-known, it was not regularly applied for the consideration of Wilson lines and their correlators.

In the line of derivation it was natural to split the problem of exponentiation onto two independent problems: the exponentiation within the non-Abelian gauge theory, and the exponentiation of a matrix object (see section 4.2). As the result of such consideration the exponentiated expression is split onto two components, those are the matrix exponentiation kernel (MEK) and the defect of exponentiation. These two components are very different both in the form and in the content. MEK contains all the essential information about the exponentiated expression. While the defect represents the difference between the matrix and the scalar exponentiation procedure, and it is an algebraic function of MEK.

In this way, the knowledge of MEK results to the knowledge of the complete exponent. The expression for MEK has the form of a sum of correlators of the operators $V$ (4.20). The operators $V$ have very peculiar form of nested commutators of gauge fields, and play an exceptional role in the non-Abelian exponentiation. Undoubtedly, further investigation should reveal exceptional properties of the operators $V$, and hence, of MEK. Good illustration of non-trivial properties of MEK is the correlator of light-like Wilson lines in MGEW approximation. In this case MEK is given exactly by one-loop diagram (see section 5.2).

The defect of exponentiation is an algebraic function of MEK. In section 4.4 we have shown that the defect is given by the simple recursive formula (4.30), where the most nontrivial operation is the procedure of the matrix symmetrization. Therefore, the evaluation of the defect can be easily automated.

In section 5, we have performed the comparison of the presented approach with existing approaches. We have shown the complete agreement between the presented approach and the standard approach to the cusp exponentiation at two-loop order [3, 4] (here, the standard approach is the approach based on the modification of color factors, which can be applied only for singlet configurations of Wilson lines). We have also demonstrated an agreement and the relation of the presented approach to the exponentiated diagrams obtained by the replica trick $[5,6]$. Remarkably, the splitting of the exponent onto MEK and the defect, can be also traced within the replica approach. Thus, we conclude that the presented approach is in complete agreement with other methods of the exponentiation.

Using MGEW approximation we have analyzed the light-like configuration of Wilson lines. In this approximation and kinematics the web diagrams can be evaluated with a minimal effort, they are given entirely by one-loop MEK and the corresponded defect. Remarkably, that MGEW part of the complete expression is gauge invariant that allows us to make a judgement about the form of the whole expression. Using this approximation we observe the violation of the dipole formula for infrared singularities of parton scattering amplitude at the three-loop order. However, we can not strictly conclude the violation of the dipole formula due to the possible cancelation of violating terms with similar terms from the diagrams do not contributing MGEW approximation.

The presented procedure of exponentiation allows further generalizations. So, for example, the correlator of ordinary product of Wilson lines (i.e. the diagrammatic series with exchanges by real particles) can be exponentiated by the same procedure, as it is demonstrated in section 3.3. Therefore, the presented exponentiation procedure can be 
applied to a wide range of physical tasks, e.g. calculation of the soft anomalous dimension, the threshold resummation, the evaluation of the soft factor for transverse momentum dependent factorization, description of diffractive processes.

\section{Acknowledgments}

I thank A.M. Moiseeva and R. Pasechnik for numerous stimulating discussions. This work is supported in part by the European Community-Research Infrastructure Integrating Activity "Study of Strongly Interacting Matter" (HadronPhysics3, Grant Agreement No. 28 3286) and the Swedish Research Council grants 621-2011-5080 and 621-2013-4287.

\section{A Operator $V_{n}$ at low orders}

In this appendix we present the explicit expression for the operators $V_{n}$ up to the fourth order. These expressions are used for the calculation in the section 5 .

The operator $V_{\gamma}^{a}$ is defined in (4.1), (4.2), while the operator $V_{n, \gamma}^{a}$ is defined in (4.6). Elaborating the matrix structure we obtain the expressions for the first four operators

$$
\begin{aligned}
V_{1}^{a}= & i g \int_{0}^{1} d \tau A_{0}^{a} \\
V_{2}^{a}= & -(i g)^{2} \int_{0}^{1} d \tau \int_{0}^{\tau} d \tau_{1} \operatorname{tr}\left(t^{a}\left[\hat{A}_{1}, \hat{A}_{0}\right]\right) \\
V_{3}^{a}= & (i g)^{3} \int_{0}^{1} d \tau \int_{0}^{\tau} d \tau_{1}\left(\frac{2}{3} \int_{0}^{\tau}-\int_{0}^{\tau_{1}}\right) d \tau_{2} \operatorname{tr}\left(t^{a}\left[\hat{A}_{1}\left[\hat{A}_{2}, \hat{A}_{0}\right]\right]\right) \\
= & \frac{(i g)^{3}}{3} \int_{0}^{1} d \tau \int_{0}^{\tau} d \tau_{1} \int_{0}^{\tau_{1}} d \tau_{2} \operatorname{tr}\left\{t^{a}\left(\left[\left[\hat{A}_{0}, \hat{A}_{1}\right], \hat{A}_{2}\right]-\left[\left[\hat{A}_{1}, \hat{A}_{2}\right], \hat{A}_{0}\right]\right)\right\} \\
V_{4}^{a}= & -(i g)^{4} \int_{0}^{1} d \tau \int_{0}^{\tau} d \tau_{1}\left(\int_{0}^{\tau_{1}} \int_{0}^{\tau_{2}}-\frac{2}{3} \int_{0}^{\tau_{1}} \int_{0}^{\tau}-\frac{2}{3} \int_{0}^{\tau} \int_{0}^{\tau_{2}}+\frac{1}{2} \int_{0}^{\tau} \int_{0}^{\tau}\right) d \tau_{2} d \tau_{3} \\
& \times \operatorname{tr}\left(t^{a}\left[\hat{A}_{1}\left[\hat{A}_{2}\left[\hat{A}_{3}, \hat{A}_{0}\right]\right]\right]\right) \\
= & -\frac{(i g)^{4}}{6} \int_{0}^{1} d \tau \int_{0}^{\tau} d \tau_{1} \int_{0}^{\tau_{1}} d \tau_{2} \int_{0}^{\tau_{2}} d \tau_{3} \\
& \times \operatorname{tr}\left\{t ^ { a } \left(\left[\left[\left[\hat{A}_{1}, \hat{A}_{2}\right] \hat{A}_{3}\right], \hat{A}_{0}\right]-\left[\left[\left[\hat{A}_{0}, \hat{A}_{1}\right] \hat{A}_{2}\right], \hat{A}_{3}\right]+\left[\left[\left[\hat{A}_{0}, \hat{A}_{3}\right] \hat{A}_{2}\right], \hat{A}_{1}\right]\right.\right. \\
& \left.\left.-\left[\left[\left[\hat{A}_{2}, \hat{A}_{3}\right] \hat{A}_{1}\right], \hat{A}_{0}\right]\right)\right\},
\end{aligned}
$$

where $\hat{A}_{i}=\dot{\gamma}^{\mu}\left(\tau_{i}\right) t_{a} A_{\mu}^{a}\left(\gamma\left(\tau_{i}\right)\right)$ and $\hat{A}_{0}=\dot{\gamma}^{\mu}(\tau) t_{a} A_{\mu}^{a}(\gamma(\tau))$. Here, as everywhere in the article, $\gamma(\tau)$ is a parametrization of a Wilson line curve, $\dot{\gamma}(\tau)$ is a tangent to the curve, and $t_{a}$ is the generator of a gauge group. The expressions for $V_{3,4}$ after the first equality symbol have the form resulting directly from eqs. (4.1), (4.2), while the expressions after the second equality symbol are obtained by rearranging the integrals and renaming the integration variables. 
The expression (A.1) is valid for the Wilson line in any representation. The representation dependence is concentrated in the generator prefactor of operator $V$ in eq. (4.2). It can be archived by the selection of the proper normalization condition for the generators and structure constants. The normalization condition used in (A.1) is

$$
\left[t^{a}, t^{b}\right]=i f^{a b c} t^{c}, \quad \operatorname{tr}\left(t^{a} t^{b}\right)=\frac{\delta^{a b}}{2},
$$

for generators of any representation.

For practical application one usually consider the straight segments of Wilson lines. Let us consider a straight Wilson line from the point $y^{\mu}$ to the point $z^{\mu}$. In this case the contour parametrization reads

$$
\gamma(\tau)=\tau z^{\mu}+(1-\tau) y^{\mu},
$$

and the tangent to the contour is the constant vector

$$
\dot{\gamma}^{\mu}(\tau)=z^{\mu}-y^{\mu}=v^{\mu} .
$$

The Feynman rules for the operators $V_{n}$ in the position space are defined as

$$
V_{a, a_{1} \ldots a_{n}}^{\mu_{1} \ldots \mu_{n}}\left(x_{1}, \ldots, x_{n}\right)=\left.\frac{\delta}{\delta A_{\mu_{1}}^{a_{1}}\left(x_{1}\right)} \cdots \frac{\delta}{\delta A_{\mu_{n}}^{a_{n}}\left(x_{n}\right)} V_{\gamma}^{a}\right|_{A=0} .
$$

Therefore, for a straight Wilson segment (A.2) the Feynman rules corresponding to operators $V_{1,2,3,4}$ read

$$
\begin{aligned}
V_{a, a_{1}}^{\mu_{1}}\left(x_{1}\right)= & i g \delta_{a a_{1}} v^{\mu_{1}} \theta_{1}, \\
V_{a, a_{1} a_{2}}^{\mu_{1} \mu_{2}}\left(x_{1}, x_{2}\right)= & \frac{i g^{2}}{2} f_{a a_{1} a_{2}} v^{\mu_{1}} v^{\mu_{2}}\left(\theta_{12}-\theta_{21}\right), \\
V_{a, a_{1} a_{2} a_{3}}^{\mu_{1} \mu_{2} \mu_{3}}\left(x_{1}, x_{2}, x_{3}\right)= & \frac{i g^{3}}{12} v^{\mu_{1}} v^{\mu_{2}} v^{\mu_{3}} \\
& \times\left[f^{a a_{1} b} f^{b a_{2} a_{3}}\left(\theta_{123}-\theta_{132}-\theta_{231}+\theta_{321}\right)+\mathcal{P}_{213}+\mathcal{P}_{312}\right], \\
V_{a, a_{1} a_{2} a_{3} a_{4}}^{\mu_{1} \mu_{2} \mu_{3} \mu_{4}}\left(x_{1}, x_{2}, x_{3}, x_{4}\right)= & \frac{i g^{4}}{12} v^{\mu_{1}} v^{\mu_{2}} v^{\mu_{3}} v^{\mu_{4}}\left[f_{a a_{1} b} f_{b a_{2} c} f_{c a_{3} a_{4}}\right. \\
& \times\left(\theta_{1234}-\theta_{1243}-\theta_{2341}+\theta_{2431}-\theta_{3214}+\theta_{3421}+\theta_{4213}-\theta_{4321}\right) \\
& +\mathcal{P}_{1324}+\mathcal{P}_{1432}+\mathcal{P}_{2134}+\mathcal{P}_{2431}+\mathcal{P}_{2341}+\mathcal{P}_{3142}+\mathcal{P}_{3412}+\mathcal{P}_{3214} \\
& \left.+\mathcal{P}_{4132}+\mathcal{P}_{4312}+\mathcal{P}_{4213}\right],
\end{aligned}
$$

where all $x_{i}$ are located on the path of Wilson line, and $\theta_{i \ldots k}=\theta\left(y<x_{i}<\ldots<x_{k}<\right.$ $z$ ) the Heaviside function ordering coordinates along the Wilson line. The symbol $\mathcal{P}_{i j k}$ stands for the first term in the bracket with permuted color indices and coordinates, i.e. $\left\{a_{1} a_{2} a_{3}\right\} \rightarrow\left\{a_{i} a_{j} a_{k}\right\}$ and $\left\{x_{1} x_{2} x_{3}\right\} \rightarrow\left\{x_{i} x_{j} x_{k}\right\}$.

In the case of half-infinite straight Wilson lines it is convenient to use the same parametrization as for the segment (A.2). The only change is that the scalar parameter runs over the infinite range $\tau \in(0, \infty)$. Such a configuration of Wilson lines can have 
additional infrared divergences, resulting from the interaction with gluons at infinity. In order to regularize these divergences one commonly uses the suppressing exponent with infinitesimal argument $[22,30]$. Formally such a regularization implies the change of the gauge field within the Wilson line

$$
A_{\mu}(v \tau+y) \rightarrow A_{\mu}(v \tau+y) e^{-i \tau \delta \sqrt{v^{2}-i \varepsilon}},
$$

where $\delta$ and $\varepsilon$ are infinitesimal and $\delta>\varepsilon>0$. Within such a regularization the largedistance singularities are regularized for space-, time and light-like Wilson lines. Thus, the change of the Feynman rules for a half-infinite Wilson lines is minimal. Namely, the function $\theta_{i \ldots k}$ should be replaced by $\theta\left(y<x_{i}<\ldots<x_{k}<\infty\right) \exp \left(-i \delta \sqrt{v^{2}-i \varepsilon} \sum_{i} x_{i}\right)$.

Open Access. This article is distributed under the terms of the Creative Commons Attribution License (CC-BY 4.0), which permits any use, distribution and reproduction in any medium, provided the original author(s) and source are credited.

\section{References}

[1] D.R. Yennie, S.C. Frautschi and H. Suura, The infrared divergence phenomena and high-energy processes, Annals Phys. 13 (1961) 379 [INSPIRE].

[2] G.F. Sterman, Infrared Divergences in Perturbative QCD, AIP Conf. Proc. 74 (1981) 22 [INSPIRE].

[3] J.G.M. Gatheral, Exponentiation of Eikonal Cross-sections in Nonabelian Gauge Theories, Phys. Lett. B 133 (1983) 90 [INSPIRE].

[4] J. Frenkel and J.C. Taylor, Nonabelian Eikonal Exponentiation, Nucl. Phys. B 246 (1984) 231 [INSPIRE].

[5] E. Laenen, G. Stavenga and C.D. White, Path integral approach to eikonal and next-to-eikonal exponentiation, JHEP 03 (2009) 054 [arXiv: 0811.2067] [INSPIRE].

[6] E. Gardi, E. Laenen, G. Stavenga and C.D. White, Webs in multiparton scattering using the replica trick, JHEP 11 (2010) 155 [arXiv:1008.0098] [INSPIRE].

[7] A. Mitov, G.F. Sterman and I. Sung, Diagrammatic Exponentiation for Products of Wilson Lines, Phys. Rev. D 82 (2010) 096010 [arXiv:1008.0099] [INSPIRE].

[8] E. Gardi, J.M. Smillie and C.D. White, The Non-Abelian Exponentiation theorem for multiple Wilson lines, JHEP 06 (2013) 088 [arXiv:1304.7040] [INSPIRE].

[9] A.A. Vladimirov, Generating function for web diagrams, Phys. Rev. D 90 (2014) 066007 [arXiv: 1406.6253] [INSPIRE].

[10] E. Gardi and C.D. White, General properties of multiparton webs: Proofs from combinatorics, JHEP 03 (2011) 079 [arXiv:1102.0756] [INSPIRE].

[11] G. Falcioni, E. Gardi, M. Harley, L. Magnea and C.D. White, Multiple Gluon Exchange Webs, JHEP 10 (2014) 010 [arXiv:1407.3477] [INSPIRE].

[12] A.N. Vasil'ev, The field theoretic renormalization group in critical behavior theory and stochastic dynamics, Chapman and Hall/CRC, Boca Raton U.S.A. (2004).

[13] A.V. Belitsky, Two loop renormalization of Wilson loop for Drell-Yan production, Phys. Lett. B 442 (1998) 307 [hep-ph/9808389] [INSPIRE]. 
[14] V.E. Nazaikinskii, V.E. Shatalov and B. Yu. Sternin, Methods of noncommutative analysis: theory and applications, New York: Walter de Gruyter, Berlin Germany (1996).

[15] A.V. Belitsky, X. Ji and F. Yuan, Final state interactions and gauge invariant parton distributions, Nucl. Phys. B 656 (2003) 165 [hep-ph/0208038] [INSPIRE].

[16] A. Grozin, J.M. Henn, G.P. Korchemsky and P. Marquard, Three Loop Cusp Anomalous Dimension in QCD, Phys. Rev. Lett. 114 (2015) 062006 [arXiv: 1409.0023] [INSPIRE].

[17] D. Knauss and K. Scharnhorst, Two Loop Renormalization of Nonsmooth String Operators in Yang-Mills Theory, Annalen Phys. 41 (1984) 331 [InSPIRE].

[18] I.A. Korchemskaya and G.P. Korchemsky, On lightlike Wilson loops, Phys. Lett. B 287 (1992) 169 [INSPIRE].

[19] G.P. Korchemsky and A.V. Radyushkin, Renormalization of the Wilson Loops Beyond the Leading Order, Nucl. Phys. B 283 (1987) 342 [InSPIRE].

[20] O. Erdoğan and G.F. Sterman, Gauge Theory Webs and Surfaces, Phys. Rev. D 91 (2015) 016003 [arXiv: 1112.4564] [INSPIRE].

[21] J.M. Henn and T. Huber, The four-loop cusp anomalous dimension in $\mathcal{N}=4$ super Yang-Mills and analytic integration techniques for Wilson line integrals, JHEP 09 (2013) 147 [arXiv: 1304.6418] [INSPIRE].

[22] E. Gardi, From Webs to Polylogarithms, JHEP 04 (2014) 044 [arXiv: 1310.5268] [INSPIRE].

[23] M. Sjödahl, ColorMath - A package for color summed calculations in $\mathrm{SU}\left(N_{c}\right)$, Eur. Phys. J. C 73 (2013) 2310 [arXiv:1211.2099] [InSPIRE].

[24] S.M. Aybat, L.J. Dixon and G.F. Sterman, The Two-loop anomalous dimension matrix for soft gluon exchange, Phys. Rev. Lett. 97 (2006) 072001 [hep-ph/0606254] [INSPIRE].

[25] S.M. Aybat, L.J. Dixon and G.F. Sterman, The Two-loop soft anomalous dimension matrix and resummation at next-to-next-to leading pole, Phys. Rev. D 74 (2006) 074004 [hep-ph/0607309] [INSPIRE].

[26] T. Becher and M. Neubert, Infrared singularities of scattering amplitudes in perturbative QCD, Phys. Rev. Lett. 102 (2009) 162001 [Erratum ibid. 111 (2013) 199905] [arXiv: 0901.0722] [INSPIRE].

[27] E. Gardi and L. Magnea, Factorization constraints for soft anomalous dimensions in QCD scattering amplitudes, JHEP 03 (2009) 079 [arXiv:0901.1091] [INSPIRE].

[28] L.J. Dixon, E. Gardi and L. Magnea, On soft singularities at three loops and beyond, JHEP 02 (2010) 081 [arXiv:0910.3653] [INSPIRE].

[29] L. Magnea, Progress on the infrared structure of multi-particle gauge theory amplitudes, PoS (LL2014) 073 [arXiv: 1408.0682] [INSPIRE].

[30] E. Gardi, J.M. Smillie and C.D. White, On the renormalization of multiparton webs, JHEP 09 (2011) 114 [arXiv:1108.1357] [INSPIRE]. 